\title{
CONDITIONING AN ADDITIVE FUNCTIONAL OF A MARKOV CHAIN TO STAY NONNEGATIVE. I. SURVIVAL FOR A LONG TIME
}

\author{
SAUL D. JACKA, *** \\ ZORANA LAZIC **** AND \\ JON WARREN, ${ }^{* * * * *}$ University of Warwick
}

\begin{abstract}
Let $\left(X_{t}\right)_{t \geq 0}$ be a continuous-time irreducible Markov chain on a finite state space $E$, let $v$ be a map $v: E \rightarrow \mathbb{R} \backslash\{0\}$, and let $\left(\varphi_{t}\right)_{t \geq 0}$ be an additive functional defined by $\varphi_{t}=\int_{0}^{t} v\left(X_{s}\right) \mathrm{d} s$. We consider the case in which the process $\left(\varphi_{t}\right)_{t \geq 0}$ is oscillating and that in which $\left(\varphi_{t}\right)_{t \geq 0}$ has a negative drift. In each of these cases, we condition the process $\left(X_{t}, \varphi_{t}\right)_{t \geq 0}$ on the event that $\left(\varphi_{t}\right)_{t \geq 0}$ is nonnegative until time $T$ and prove weak convergence of the conditioned process as $T \rightarrow \infty$.
\end{abstract}

Keywords: Markov chain; conditional law; weak convergence; survival

2000 Mathematics Subject Classification: Primary 60J27

Secondary 60B 10

\section{Introduction}

The problem of conditioning a stochastic process to remain forever in a certain region has been extensively studied in the literature. Many authors have addressed essentially the same problem by conditioning a process with a possibly finite lifetime to live forever. An interesting case is when the event that the process remains in some region is of probability 0 , or, in terms of the lifetime of the process restricted to the region, when the process has a finite lifetime with probability 1 . In that case, the process cannot be conditioned to stay in the region forever in the standard way. Instead, this conditioning can be approximated by conditioning the process to stay in the region for a long time (i.e. for a sequence of times tending to infinity).

There are many well-known examples of such conditionings in which weak convergence of the approximating process occurs. For instance, Knight (1969) showed that the standard Brownian motion conditioned not to cross 0 for a long time converges weakly to a threedimensional Bessel process; Iglehart (1974) considered a general random walk conditioned to stay nonnegative for a long time and showed that it converges weakly; Pinsky (1985) showed that, under certain conditions, a homogeneous diffusion on $\mathbb{R}^{d}$ conditioned to remain in an open connected bounded region for a long time converges weakly to a homogeneous diffusion; and Jacka and Roberts (1988) proved weak convergence of an Itô diffusion conditioned to remain in an interval $(a, b)$ for a long time.

However, weak convergence of the approximations does not always occur. There are counterexamples in which a process conditioned to stay in a region for a long time either

Received 21 April 2004; revision received 31 May 2005.

* Postal address: Department of Statistics, University of Warwick, Coventry CV4 7AL, UK.

** Email address: s.d.jacka@warwick.ac.uk

*** Email address: z.lazic@warwick.ac.uk

**** Email address: j.warren@warwick.ac.uk 
does not converge at all or does so, but to a dishonest limit. Bertoin and Doney (1994) and Jacka and Warren (2002) gave examples of such processes.

In this paper, we are concerned with another example of the conditioning of a process to stay in a region. We consider a finite-state space, continuous-time Markov chain $\left(X_{t}\right)_{t \geq 0}$ and an associated fluctuating additive functional $\left(\varphi_{t}\right)_{t \geq 0}$. The aim is to condition the Markov process $\left(X_{t}, \varphi_{t}\right)_{t \geq 0}$ on the event that the fluctuating functional stays nonnegative.

The process $\left(\varphi_{t}\right)_{t \geq 0}$ has three possible types of behaviour, in two of which - when it oscillates and when it drifts to negative infinity - the event that it stays nonnegative is of probability 0 . We are interested in performing conditioning in these two cases.

A similar question was discussed in Bertoin and Doney (1994) for a real-valued random walk. It was shown there that, under certain conditions, an oscillating random walk or a random walk with a negative drift, conditioned to stay nonnegative for a long time, converges weakly to an honest limit which is an $h$-transform of the original random walk killed when it hits 0 . This work presents the analogous result for the process $\left(X_{t}, \varphi_{t}\right)_{t \geq 0}$.

The organization of the paper is as follows: the exact formulation of the problem and the main results are given in Section 2, the notation and preliminary results used in the proofs of the main theorems are given in Section 3, the proof of the result in the oscillating case is given is Section 4, and the proof of the result in the negative-drift case is given in Section 5.

\section{The problem and main results}

Let $\left(X_{t}\right)_{t \geq 0}$ be an irreducible honest Markov chain on a finite state space $E$. Let $v$ be a map $v: E \rightarrow \mathbb{R} \backslash\{0\}$ and suppose that both $E^{+}=v^{-1}(0, \infty)$ and $E^{-}=v^{-1}(-\infty, 0)$ are nonempty.

Define the process $\left(\varphi_{t}\right)_{t \geq 0}$ by

$$
\varphi_{t}=\varphi+\int_{0}^{t} v\left(X_{s}\right) \mathrm{d} s, \quad \varphi \in \mathbb{R} .
$$

For any $y \in \mathbb{R}$, let

$$
E_{y}^{+}=(E \times(y, \infty)) \cup\left(E^{+} \times\{y\}\right)
$$

and let $H_{0}=\inf \left\{t>0: \varphi_{t}<0\right\}$. The aim is to condition the process $\left(X_{t}, \varphi_{t}\right)_{t \geq 0}$, starting in $E_{0}^{+}$, on the event $\left\{H_{0}=\infty\right\}$.

There are three possible cases, depending on the behaviour of the process $\left(\varphi_{t}\right)_{t \geq 0}$. When the process $\left(\varphi_{t}\right)_{t \geq 0}$ drifts to $\infty$, the event $\left\{H_{0}=\infty\right\}$ is of positive probability, which implies that the conditioning of the process $\left(X_{t}, \varphi_{t}\right)_{t \geq 0}$ on it can be performed in the standard way. However, when the process $\left(\varphi_{t}\right)_{t \geq 0}$ oscillates or drifts to $-\infty$, the event $\left\{H_{0}=\infty\right\}$ is of probability 0 , and the conditioning of $\left(X_{t}, \varphi_{t}\right)_{t \geq 0}$ on it cannot be performed in the standard way. We concentrate on the two latter cases and define the conditioning of $\left(X_{t}, \varphi_{t}\right)_{t \geq 0}$ on $\left\{H_{0}=\infty\right\}$ as the limit as $T \rightarrow \infty$ of the conditioning of $\left(X_{t}, \varphi_{t}\right)_{t \geq 0}$ on $\left\{H_{0}>t\right\}$.

Let $\mathrm{P}_{(e, \varphi)}$ denote the law of the process $\left(X_{t}, \varphi_{t}\right)_{t \geq 0}$ starting at $(e, \varphi)$, and let $\mathrm{E}_{(e, \varphi)}$ denote the expectation operator associated with $\mathrm{P}_{(e, \varphi)}$. Let $\mathrm{P}_{(e, \varphi)}^{(T)}, T>0$, denote the law of the process $\left(X_{t}, \varphi_{t}\right)_{t \geq 0}$ starting at $(e, \varphi) \in E_{0}^{+}$and conditioned on $\left\{H_{0}>T\right\}$, and let $\left(\mathcal{F}_{t}\right)_{t \geq 0}$ be the natural filtration of $\left(X_{t}\right)_{t \geq 0}$. We are interested in the weak convergence of the restrictions of $\left(\mathrm{P}_{(e, \varphi)}^{(T)}\right)_{T \geq 0}$ to $\mathcal{F}_{t}$ as $T \rightarrow \infty$.

Let $\boldsymbol{Q}$ denote the conservative irreducible $Q$-matrix of the process $\left(X_{t}\right)_{t \geq 0}$ and let $\boldsymbol{V}$ be the diagonal matrix $\operatorname{diag}(v(e)), e \in E$. Let $\boldsymbol{V}^{-1} \boldsymbol{Q} \boldsymbol{\Gamma}=\boldsymbol{\Gamma} \boldsymbol{G}$ be the unique Wiener-Hopf 
factorization of the matrix $\boldsymbol{V}^{-1} \boldsymbol{Q}$ (see Barlow et al. (1980) or refer to Lemma 3.4, below). Let $\boldsymbol{J}, \boldsymbol{J}_{1}$, and $\boldsymbol{J}_{2}$ be the matrices

$$
\boldsymbol{J}=\left(\begin{array}{cc}
\boldsymbol{I} & \mathbf{0} \\
\mathbf{0} & -\boldsymbol{I}
\end{array}\right), \quad \boldsymbol{J}_{1}=\left(\begin{array}{cc}
\boldsymbol{I} & \mathbf{0} \\
\mathbf{0} & \mathbf{0}
\end{array}\right), \quad \boldsymbol{J}_{2}=\left(\begin{array}{cc}
\mathbf{0} & \mathbf{0} \\
\mathbf{0} & \boldsymbol{I}
\end{array}\right),
$$

and let the matrix $\boldsymbol{\Gamma}_{2}$ be given by $\boldsymbol{\Gamma}_{2}=\boldsymbol{J} \boldsymbol{\Gamma} \boldsymbol{J}$. Throughout, $\boldsymbol{I}$ denotes the identity matrix, and $M(i, j)=[\boldsymbol{M}](i, j)$ is used to denote the $(i, j)$ th entry of a matrix $\boldsymbol{M}$. In $\boldsymbol{J}_{1}$ and the first row of $\boldsymbol{J}, \boldsymbol{I}$ is of order $\left|E^{+}\right|$, while in $\boldsymbol{J}_{2}$ and the second row of $\boldsymbol{J}$, it is of order $\left|E^{-}\right|$.

We now state our main result in the oscillating case.

Theorem 2.1. Suppose that the process $\left(\varphi_{t}\right)_{t \geq 0}$ oscillates. Then, for a fixed $(e, \varphi) \in E_{0}^{+}$and $t \geq 0$, the restrictions of the measures $\left(\mathrm{P}_{(e, \varphi)}^{(T)}\right)_{T \geq 0}$ to $\mathcal{F}_{t}$ converge weakly, as $T \rightarrow \infty$, to the restriction of the probability measure $\mathrm{P}_{(e, \varphi)}^{h_{r}}$ defined by

$$
\mathrm{P}_{(e, \varphi)}^{h_{\boldsymbol{r}}}(A)=\frac{\mathrm{E}_{(e, \varphi)}\left(1(A) h_{\boldsymbol{r}}\left(X_{s}, \varphi_{s}\right) 1\left(s<H_{0}\right)\right)}{h_{\boldsymbol{r}}(e, \varphi)}, \quad s \geq 0, A \in \mathcal{F}_{s},
$$

where $h_{r}(e, y)$ is a positive, harmonic function for the process $\left(X_{t}, \varphi_{t}\right)_{t \geq 0}$ given by $h_{\boldsymbol{r}}(e, y)=$ $\left[\mathrm{e}^{-y \boldsymbol{V}^{-1}} \boldsymbol{Q} \boldsymbol{J}_{1} \boldsymbol{\Gamma}_{2} \boldsymbol{r}\right](e),(e, y) \in E \times \mathbb{R}$, for a vector $\boldsymbol{r}$ satisfying $\boldsymbol{V}^{-1} \boldsymbol{Q r}=\mathbf{1}$, and $1(S)$ denotes the indicator function of a set $S$.

Let $\beta_{0}$ be the point at which the Perron-Frobenius eigenvalue $\alpha(\beta)$ of the matrix $\boldsymbol{Q}-\beta \boldsymbol{V}$, $\beta \in \mathbb{R}$, attains its global minimum (see Lemma 3.9, below). Let $\alpha_{0}=\alpha\left(\beta_{0}\right)$ and $\boldsymbol{g}_{0}$ be the Perron-Frobenius eigenvalue and right eigenvector, respectively, of the matrix $\boldsymbol{Q}-\beta_{0} \boldsymbol{V}$, and let $\boldsymbol{G}_{0}$ be the diagonal matrix $\operatorname{diag}\left(\boldsymbol{g}_{0}\right)$. Let $\boldsymbol{Q}^{0}$ be the $|E| \times|E|$ matrix with entries

$$
Q^{0}\left(e, e^{\prime}\right)=\left[\boldsymbol{G}_{0}^{-1}\left(\boldsymbol{Q}-\alpha_{0} \boldsymbol{I}-\beta_{0} \boldsymbol{V}\right) \boldsymbol{G}_{0}\right]\left(e, e^{\prime}\right), \quad e, e^{\prime} \in E .
$$

As we shall see later, the matrix $Q^{0}$ is a conservative irreducible $Q$-matrix (see Lemma 3.11, below). Let $\boldsymbol{V}^{-1} \boldsymbol{Q}^{0} \boldsymbol{\Gamma}^{0}=\boldsymbol{\Gamma}^{0} \boldsymbol{G}^{0}$ be the unique Wiener-Hopf factorization of the matrix $\boldsymbol{V}^{-1} \boldsymbol{Q}^{0}$ and let $\Gamma_{2}^{0}=J \Gamma^{0} J$. We can now state our main result in the negative-drift case.

Theorem 2.2. Suppose that the process $\left(\varphi_{t}\right)_{t \geq 0}$ drifts to $-\infty$. For fixed $(e, \varphi),\left(e^{\prime}, \varphi^{\prime}\right) \in E_{0}^{+}$ and $t \geq 0$, if all nonzero eigenvalues of the matrix $\boldsymbol{V}^{-1} Q^{0}$ are simple and if

$$
\lim _{T \rightarrow \infty} \frac{\mathrm{P}_{\left(e^{\prime}, \varphi^{\prime}\right)}\left(H_{0}>T-t\right)}{\mathrm{P}_{(e, \varphi)}\left(H_{0}>T\right)}
$$

exists, then the restrictions of the measures $\left(\mathrm{P}_{(e, \varphi)}^{(T)}\right)_{T \geq 0}$ to $\mathcal{F}_{t}$ converge weakly, as $T \rightarrow \infty$, to the restriction of a probability measure $\mathrm{P}_{(e, \varphi)}^{h^{0}}$ that is defined by

$$
\mathrm{P}_{(e, \varphi)}^{h^{r^{0}}}(A)=\frac{\mathrm{E}_{(e, \varphi)}\left(1(A) h_{\boldsymbol{r}^{0}}\left(X_{s}, \varphi_{s}, t\right) 1\left(s<H_{0}\right)\right)}{h_{\boldsymbol{r}^{0}}(e, \varphi, s)}, \quad s \geq 0, A \in \mathcal{F}_{s},
$$

where $h_{r^{0}}(e, y, t)$ is a positive, space-time-harmonic function for the process $\left(X_{t}, \varphi_{t}\right)_{t \geq 0}$ given by $h_{\boldsymbol{r}^{0}}(e, y, t)=\mathrm{e}^{-\alpha_{0} t} \mathrm{e}^{-\beta_{0} y}\left[\boldsymbol{G}_{0} \mathrm{e}^{-y \boldsymbol{V}^{-1} \boldsymbol{Q}^{0}} \boldsymbol{J}_{1} \boldsymbol{\Gamma}_{2}^{0} \boldsymbol{r}^{0}\right](e), \quad(e, y, t) \in E \times \mathbb{R} \times[0, \infty)$, and $\boldsymbol{V}^{-1} \boldsymbol{Q}^{0} \boldsymbol{r}^{0}=\mathbf{1}$.

Note that $\mathrm{P}_{(e, \varphi)}^{h_{r}}$ and $\mathrm{P}_{(e, \varphi)}^{h_{r 0}}$ are $h$-transforms of the transition kernel for the process $\left(X_{t}, \varphi_{t}\right)_{t \geq 0}$ killed when the process $\left(\varphi_{t}\right)_{t \geq 0}$ crosses 0 . 


\section{Notation and preliminary results}

The purpose of this section is to introduce notation, recall some results, and prove some others that are needed for the proofs in the subsequent two sections. The proofs are fairly straightforward and are included for the sake of completeness.

Lemma 3.1. Let $\boldsymbol{Q}$ be an irreducible essentially nonnegative matrix, let $\boldsymbol{V}$ be a diagonal matrix, and let $\beta \in \mathbb{R}$. Then the matrix $\boldsymbol{Q}-\beta \boldsymbol{V}$ is also an irreducible essentially nonnegative matrix.

Proof. The proof follows directly from the definition of an irreducible essentially nonnegative matrix (see Seneta (1981)).

The following three lemmas were proved in Barlow et al. (1980). We state them here in the notation we intend to use.

Lemma 3.2. For a fixed $\alpha>0$, there exist a unique pair $\left(\boldsymbol{\Pi}_{\alpha}^{+}, \boldsymbol{\Pi}_{\alpha}^{-}\right)$, where $\Pi_{\alpha}^{+}$is a $\left|E^{-}\right| \times\left|E^{+}\right|$ matrix and $\Pi_{\alpha}^{-}$is a $\left|E^{+}\right| \times\left|E^{-}\right|$matrix, and $Q$-matrices $\boldsymbol{G}_{\alpha}^{+}$and $\boldsymbol{G}_{\alpha}^{-}$on $E^{+} \times E^{+}$and $E^{-} \times E^{-}$, respectively, such that if

$$
\boldsymbol{\Gamma}_{\alpha}=\left(\begin{array}{cc}
\boldsymbol{I} & \boldsymbol{\Pi}_{\alpha^{-}} \\
\boldsymbol{\Pi}_{\alpha}^{+} & \boldsymbol{I}
\end{array}\right) \quad \text { and } \quad \boldsymbol{G}_{\alpha}=\left(\begin{array}{cc}
\boldsymbol{G}_{\alpha}^{+} & \mathbf{0} \\
\mathbf{0} & -\boldsymbol{G}_{\alpha}^{-}
\end{array}\right),
$$

then $\boldsymbol{\Gamma}_{\alpha}$ is invertible and $\boldsymbol{\Gamma}_{\alpha}^{-1} \boldsymbol{V}^{-1}(\boldsymbol{Q}-\alpha \boldsymbol{I}) \boldsymbol{\Gamma}_{\alpha}=\boldsymbol{G}_{\alpha}$. Moreover, $\boldsymbol{\Pi}_{\alpha}^{+}$and $\boldsymbol{\Pi}_{\alpha}^{-}$are strictly substochastic.

Recall that the subspace $E_{y}^{+}, y \in \mathbb{R}$, is given by $E_{y}^{+}=(E \times(y, \infty)) \cup\left(E^{+} \times\{y\}\right)$. Let $E_{y}^{-}, y \in \mathbb{R}$, be the subspace $E_{y}^{-}=(E \times(-\infty, y)) \cup\left(E^{-} \times\{y\}\right)$, and let $H_{y}, y \in \mathbb{R}$, be the first crossing time of the level $y$ by the process $\left(\varphi_{t}\right)_{t \geq 0}$, defined by

$$
H_{y}= \begin{cases}\inf \left\{t>0: \varphi_{t}<y\right\} & \text { if }\left(X_{t}, \varphi_{t}\right)_{t \geq 0} \operatorname{startsin} E_{y}^{+} \\ \inf \left\{t>0: \varphi_{t}>y\right\} & \text { if }\left(X_{t}, \varphi_{t}\right)_{t \geq 0} \operatorname{startsin} E_{y}^{-}\end{cases}
$$

Lemma 3.3. Let $\alpha>0$ be fixed. Then

$$
\begin{aligned}
\mathrm{E}_{(e, 0)}\left(\mathrm{e}^{-\alpha H_{0}} 1\left(X_{H_{0}}=e^{\prime}\right)\right) & =\Pi_{\alpha}^{+}\left(e, e^{\prime}\right), & & \left(e, e^{\prime}\right) \in E^{-} \times E^{+}, \\
\mathrm{E}_{(e, 0)}\left(\mathrm{e}^{-\alpha H_{0}} 1\left(X_{H_{0}}=e^{\prime}\right)\right) & =\Pi_{\alpha}^{-}\left(e, e^{\prime}\right), & & \left(e, e^{\prime}\right) \in E^{+} \times E^{-}, \\
\mathrm{E}_{(e, 0)}\left(\mathrm{e}^{-\alpha H_{y}} 1\left(X_{H_{y}}=e^{\prime}\right)\right) & =\left[\mathrm{e}^{y \boldsymbol{G}_{\alpha}^{+}}\right]\left(e, e^{\prime}\right), & & \left(e, e^{\prime}\right) \in E^{+} \times E^{+}, y>0, \\
\mathrm{E}_{(e, 0)}\left(\mathrm{e}^{-\alpha H_{-y}} 1\left(X_{H_{-y}}=e^{\prime}\right)\right) & =\left[\mathrm{e}^{y \boldsymbol{G}_{\alpha}^{-}}\right]\left(e, e^{\prime}\right), & & \left(e, e^{\prime}\right) \in E^{-} \times E^{-}, y>0 .
\end{aligned}
$$

Lemma 3.4. There exist a unique pair $\left(\Pi^{+}, \Pi^{-}\right)$, where $\Pi^{+}$is a $\left|E^{-}\right| \times\left|E^{+}\right|$matrix and $\Pi^{-}$ is a $\left|E^{+}\right| \times\left|E^{-}\right|$matrix, and $Q$-matrices $\boldsymbol{G}^{+}$on $E^{+} \times E^{+}$and $\boldsymbol{G}^{-}$on $E^{-} \times E^{-}$such that

$$
V^{-1} Q \Gamma=\Gamma G
$$

where

$$
\boldsymbol{\Gamma}=\left(\begin{array}{cc}
\boldsymbol{I} & \boldsymbol{\Pi}^{-} \\
\boldsymbol{\Pi}^{+} & \boldsymbol{I}
\end{array}\right) \quad \text { and } \quad \boldsymbol{G}=\left(\begin{array}{cc}
\boldsymbol{G}^{+} & \mathbf{0} \\
\mathbf{0} & -\boldsymbol{G}^{-}
\end{array}\right)
$$


Moreover, $\Pi^{+}$and $\Pi^{-}$are substochastic and

$$
\begin{aligned}
\mathrm{P}_{(e, 0)}\left(X_{H_{0}}=e^{\prime}\right) & =\Pi^{+}\left(e, e^{\prime}\right), & & \left(e, e^{\prime}\right) \in E^{-} \times E^{+}, \\
\mathrm{P}_{(e, 0)}\left(X_{H_{0}}=e^{\prime}\right) & =\Pi^{-}\left(e, e^{\prime}\right), & & \left(e, e^{\prime}\right) \in E^{+} \times E^{-}, \\
\mathrm{P}_{(e, 0)}\left(X_{H_{y}}=e^{\prime}\right) & =\left[\mathrm{e}^{y G^{+}}\right]\left(e, e^{\prime}\right), & & \left(e, e^{\prime}\right) \in E^{+} \times E^{+}, y \geq 0, \\
\mathrm{P}_{(e, 0)}\left(X_{H_{-y}}=e^{\prime}\right) & =\left[\mathrm{e}^{y G^{-}}\right]\left(e, e^{\prime}\right), & & \left(e, e^{\prime}\right) \in E^{-} \times E^{-}, y \geq 0 .
\end{aligned}
$$

Lemmas 3.2 and 3.4 are said to yield the Wiener-Hopf factorizations of the matrices $\boldsymbol{V}^{-1}(\boldsymbol{Q}-\alpha \boldsymbol{I}), \alpha>0$, and $\boldsymbol{V}^{-1} \boldsymbol{Q}$, respectively.

The statements in the following lemma can easily be deduced from Lemmas 3.2, 3.3, and 3.4, so we omit their proofs.

Lemma 3.5. (i) The matrices $\Pi^{+}$and $\Pi^{-}$are positive.

(ii) If at least one of the matrices $\Pi^{+}$and $\Pi^{-}$is strictly substochastic then the matrices $\boldsymbol{I}-\boldsymbol{\Pi}^{-} \boldsymbol{\Pi}^{+}, \boldsymbol{I}-\boldsymbol{\Pi}^{+} \boldsymbol{\Pi}^{-}$, and $\boldsymbol{\Gamma}$ are invertible and

$$
\boldsymbol{\Gamma}^{-1}=\left(\begin{array}{cc}
\left(\boldsymbol{I}-\boldsymbol{\Pi}^{-} \boldsymbol{\Pi}^{+}\right)^{-1} & -\boldsymbol{\Pi}^{-}\left(\boldsymbol{I}-\boldsymbol{\Pi}^{+} \boldsymbol{\Pi}^{-}\right)^{-1} \\
-\boldsymbol{\Pi}^{+}\left(\boldsymbol{I}-\boldsymbol{\Pi}^{-} \boldsymbol{\Pi}^{+}\right)^{-1} & \left(\boldsymbol{I}-\boldsymbol{\Pi}^{+} \boldsymbol{\Pi}^{-}\right)^{-1}
\end{array}\right) .
$$

(iii) The matrices $\boldsymbol{G}^{+}$and $\boldsymbol{G}^{-}$are irreducible $Q$-matrices.

(iv) $\boldsymbol{G}^{+}$and $\boldsymbol{G}^{-}$are conservative if and only if $\boldsymbol{\Pi}^{+}$and $\boldsymbol{\Pi}^{-}$are stochastic, respectively.

(v) $\lim _{\alpha \rightarrow 0} \boldsymbol{\Gamma}_{\alpha}=\boldsymbol{\Gamma}$.

(vi) For any $y>0$ and $(e, \varphi) \in E_{0}^{+} \cap E_{y}^{-}$, or any $y<0$ and $(e, \varphi) \in E_{0}^{-} \cap E_{y}^{+}$,

$$
\mathrm{P}_{(e, \varphi)}\left(X_{H_{y}}=e^{\prime}, H_{y}<H_{0}\right)>0 \text { and } 0<\mathrm{P}_{(e, \varphi)}\left(H_{y}<H_{0}\right)<1 .
$$

(vii) For any $(e, \varphi) \in E_{0}^{+}$and $e^{\prime} \in E^{-}$, or any $(e, \varphi) \in E_{0}^{-}$and $e^{\prime} \in E^{+}$,

$$
\mathrm{P}_{(e, \varphi)}\left(X_{H_{0}}=e^{\prime}, H_{0}<\infty\right)>0 .
$$

(viii) For any $(e, \varphi) \in E \times \mathbb{R}$ and $T>0, \mathrm{P}_{(e, \varphi)}\left(H_{0}>T\right)>0$.

We now introduce vector notation that will be used in the sequel. For any vector $\boldsymbol{g}$ on $E$, let $\boldsymbol{g}^{+}$and $\boldsymbol{g}^{-}$denote its restrictions to $E^{+}$and $E^{-}$, respectively. We write the column vector $\boldsymbol{g}$ as

$$
g=\left(\begin{array}{l}
g^{+} \\
g^{-}
\end{array}\right)
$$

and the row vector $\boldsymbol{\mu}$ as $\boldsymbol{\mu}=\left(\boldsymbol{\mu}^{+}, \boldsymbol{\mu}^{-}\right)$.

It follows from Lemmas 3.2, 3.3, and 3.4 (see Barlow et al. (1980)) that the matrix $\boldsymbol{V}^{-1}(\boldsymbol{Q}-\alpha \boldsymbol{I})$ cannot have strictly imaginary eigenvalues, and that there exists a basis $\mathscr{B}(\alpha)$ in the space of all vectors on $E$ such that if $\boldsymbol{g}(\alpha)$ is in $\mathcal{B}(\alpha)$, then

$$
\left(\boldsymbol{V}^{-1}(\boldsymbol{Q}-\alpha \boldsymbol{I})-\lambda(\alpha) \boldsymbol{I}\right)^{k} \boldsymbol{g}(\alpha)=\mathbf{0},
$$

for some eigenvalue $\lambda(\alpha)$ of $\boldsymbol{V}^{-1}(\boldsymbol{Q}-\alpha \boldsymbol{I})$ and some $k \in \mathbb{N}$. The number of vectors in the basis $\mathcal{B}(\alpha)$ associated with the same eigenvalue is equal to the algebraic multiplicity of that 
eigenvalue. Let $\mathcal{N}(\alpha)$ and $\mathcal{P}(\alpha)$ be the sets of vectors $\boldsymbol{g}(\alpha) \in \mathscr{B}(\alpha)$ associated with eigenvalues with positive and with negative real parts, respectively. Then

$$
\boldsymbol{g}(\alpha) \in \mathcal{N}(\alpha) \Rightarrow \boldsymbol{g}(\alpha)=\left(\begin{array}{c}
\boldsymbol{g}^{+}(\alpha) \\
\boldsymbol{\Pi}_{\alpha}^{+} \boldsymbol{g}^{+}(\alpha)
\end{array}\right), \quad \boldsymbol{g}(\alpha) \in \mathcal{P}(\alpha) \Rightarrow \boldsymbol{g}(\alpha)=\left(\begin{array}{c}
\boldsymbol{\Pi}_{\alpha}^{-} \boldsymbol{g}^{-}(\alpha) \\
\boldsymbol{g}^{-}(\alpha)
\end{array}\right)
$$

The sets $\mathcal{N}(\alpha)$ and $\mathcal{P}(\alpha)$ contain exactly $\left|E^{+}\right|$and, respectively, $\left|E^{-}\right|$vectors and the vectors $\boldsymbol{g}^{+}(\alpha)$ and $\boldsymbol{g}^{-}(\alpha)$, for all $\boldsymbol{g}(\alpha) \in \mathcal{N}(\alpha)$ and, respectively, $\boldsymbol{g}(\alpha) \in \mathcal{P}(\alpha)$ ), form a basis in the space of all vectors on $E^{+}$and, respectively, $E^{-}$. The eigenvalues of $\boldsymbol{V}^{-1}(\boldsymbol{Q}-\alpha \boldsymbol{I})$ with strictly negative and with strictly positive real parts coincide with the eigenvalues of $\boldsymbol{G}_{\alpha}^{+}$and, respectively, $-\boldsymbol{G}_{\alpha}^{-}$.

The Wiener-Hopf factorization (3.1) of the matrix $\boldsymbol{V}^{-1} \boldsymbol{Q}$ implies that

$$
\begin{array}{lll}
\boldsymbol{G}^{+} \boldsymbol{f}^{+}=\alpha \boldsymbol{f}^{+} \quad \text { if and only if } & \boldsymbol{V}^{-1} \boldsymbol{Q}\left(\begin{array}{c}
\boldsymbol{f}^{+} \\
\boldsymbol{\Pi}^{+} \boldsymbol{f}^{+}
\end{array}\right)=\alpha\left(\begin{array}{c}
\boldsymbol{f}^{+} \\
\boldsymbol{\Pi}^{+} \boldsymbol{f}^{+}
\end{array}\right), \\
\boldsymbol{G}^{-} \boldsymbol{g}^{-}=-\beta \boldsymbol{g}^{-} \quad \text { if and only if } & \boldsymbol{V}^{-1} \boldsymbol{Q}\left(\begin{array}{c}
\boldsymbol{\Pi}^{-} \boldsymbol{g}^{-} \\
\boldsymbol{g}^{-}
\end{array}\right)=\beta\left(\begin{array}{c}
\boldsymbol{\Pi}^{-} \boldsymbol{g}^{-} \\
\boldsymbol{g}^{-}
\end{array}\right) .
\end{array}
$$

Let $\alpha_{j}, j=1, \ldots, n$, be the (not necessarily distinct) eigenvalues of the matrix $\boldsymbol{G}^{+}$, and let $-\beta_{k}, k=1, \ldots, m$, be the (not necessarily distinct) eigenvalues of the matrix $\boldsymbol{G}^{-}$. By Lemma 3.5(iii), $\boldsymbol{G}^{+}$and $\boldsymbol{G}^{-}$are irreducible $Q$-matrices, which implies that

$$
\alpha_{\max }:=\max _{1 \leq j \leq n} \operatorname{Re}\left(\alpha_{j}\right) \leq 0 \text { and }-\beta_{\min }:=\max _{1 \leq k \leq m} \operatorname{Re}\left(-\beta_{k}\right)=-\min _{1 \leq k \leq m} \operatorname{Re}\left(\beta_{k}\right) \leq 0
$$

are simple eigenvalues of $\boldsymbol{G}^{+}$and $\boldsymbol{G}^{-}$, respectively. Hence, it follows from (3.3) that all eigenvalues of $\boldsymbol{V}^{-1} \boldsymbol{Q}$ with negative and positive real parts respectively coincide with the eigenvalues of $\boldsymbol{G}^{+}$and $-\boldsymbol{G}^{-}$.

By Jordan normal form theory, there exists a basis $\mathcal{B}$, in the space of all vectors on $E$, containing exactly $n=\left|E^{+}\right|$vectors, $f_{1}, f_{2}, \ldots, f_{n}$, such that each $f_{j}, j=1, \ldots, n$, is associated with an eigenvalue $\alpha_{j}$ of $\boldsymbol{V}^{-1} \boldsymbol{Q}$ for which $\operatorname{Re}\left(\alpha_{j}\right) \leq 0$, and containing exactly $m=\left|E^{-}\right|$vectors, $\boldsymbol{g}_{1}, \boldsymbol{g}_{2}, \ldots, \boldsymbol{g}_{m}$, such that each $\boldsymbol{g}_{k}, k=1, \ldots, m$, is associated with an eigenvalue $\beta_{k}$ of $\boldsymbol{V}^{-1} \boldsymbol{Q}$ for which $\operatorname{Re}\left(\beta_{k}\right) \geq 0$. The vectors $\boldsymbol{f}_{1}^{+}, \boldsymbol{f}_{2}^{+}, \ldots, \boldsymbol{f}_{n}^{+}$form a basis $\mathcal{N}^{+}$in the space of all vectors on $E^{+}$and the vectors $\boldsymbol{g}_{1}^{-}, \boldsymbol{g}_{2}^{-}, \ldots, \boldsymbol{g}_{m}^{-}$form a basis $\mathcal{P}^{-}$in the space of all vectors on $E^{-}$.

Let $\boldsymbol{f}_{\max }$ and $\boldsymbol{g}_{\min }$ be the eigenvectors of $\boldsymbol{V}^{-1} \boldsymbol{Q}$ associated with the eigenvalues $\alpha_{\max }$ and $\beta_{\min }$, respectively. Then $\boldsymbol{f}_{\max }^{+}$and $\boldsymbol{g}_{\min }^{-}$are the Perron-Frobenius eigenvectors of the matrices $\boldsymbol{G}^{+}$and $\boldsymbol{G}^{-}$, respectively.

Lemma 3.6. (i) The vectors $\boldsymbol{f}_{\max }$ and $\boldsymbol{g}_{\min }$ are the only positive eigenvectors of the matrix $\boldsymbol{V}^{-1} \boldsymbol{Q}$.

(ii) There are no nonnegative vectors on $E^{+}$or $E^{-}$that are linearly independent of the vector $\boldsymbol{f}_{\max }^{+}$or $\boldsymbol{g}_{\min }^{-}$, respectively.

Proof. (i) Let $\boldsymbol{f}$ be a positive eigenvector of the matrix $\boldsymbol{V}^{-1} \boldsymbol{Q}$. Then, by (3.3), either $\boldsymbol{f}^{+}$ is an eigenvector of $\boldsymbol{G}^{+}$or $\boldsymbol{f}^{-}$is an eigenvector of $\boldsymbol{G}^{-}$. The only positive eigenvectors of $\boldsymbol{G}^{+}$ and $\boldsymbol{G}^{-}$are $\boldsymbol{f}_{\max }^{+}$and $\boldsymbol{g}_{\min }^{-}$, respectively. Hence, either

$$
\boldsymbol{f}=\left(\begin{array}{c}
\boldsymbol{f}_{\max }^{+} \\
\boldsymbol{\Pi}^{+} \boldsymbol{f}_{\max }^{+}
\end{array}\right)=\boldsymbol{f}_{\max } \text { or } \quad \boldsymbol{f}=\left(\begin{array}{c}
\boldsymbol{\Pi}^{-} \boldsymbol{g}_{\min }^{-} \\
\boldsymbol{g}_{\min }^{-}
\end{array}\right)=\boldsymbol{g}_{\min }
$$


Since, by Lemma 3.5(i), the matrices $\boldsymbol{\Pi}^{+}$and $\boldsymbol{\Pi}^{-}$are positive, we see that $\boldsymbol{f}_{\max }$ and $\boldsymbol{g}_{\min }$ are positive, which completes the proof.

(ii) Let $f^{+}$be a nonnegative vector on $E^{+}$independent of $f_{\max }^{+}$. Since

$$
\mathcal{N}^{+}=\left\{f_{1}^{+}, f_{2}^{+}, \ldots, f_{n}^{+}\right\}
$$

is a basis in the space of all vectors on $E^{+}$, the vector $f^{+}$has a decomposition

$$
\boldsymbol{f}^{+}=\sum_{\left\{j=1, \ldots, n: \boldsymbol{f}_{j}^{+} \neq \boldsymbol{f}_{\max }^{+}\right\}} a_{j} \boldsymbol{f}_{j}^{+},
$$

for some coefficients $a_{j}, j=1, \ldots, n$.

Let $\boldsymbol{f}_{\max }^{1,+}$ be the left Perron-Frobenius eigenvector of $\boldsymbol{G}^{+}$. Then $\boldsymbol{f}_{\max }^{1,+} \mathrm{e}^{t \boldsymbol{G}^{+}}=\mathrm{e}^{\alpha_{\max } t} \boldsymbol{f}_{\max }^{1,+}$ and $\boldsymbol{f}_{\max }^{1,+} \boldsymbol{f}_{j}^{+}=0$ for all $\boldsymbol{f}_{j}^{+} \neq \boldsymbol{f}_{\max }^{+}, j=1, \ldots, n$. Thus, for any $t \geq 0$,

$$
\boldsymbol{f}_{\max }^{1,+} \mathrm{e}^{t \boldsymbol{G}^{+}} \boldsymbol{f}^{+}=\sum_{\left\{j=1, \ldots, n: \boldsymbol{f}_{j}^{+} \neq \boldsymbol{f}_{\max }^{+}\right\}} a_{j} \boldsymbol{f}_{\max }^{1,+} \mathrm{e}^{t \boldsymbol{G}^{+}} \boldsymbol{f}_{j}^{+}=\sum_{\left\{j=1, \ldots, n: \boldsymbol{f}_{j}^{+} \neq \boldsymbol{f}_{\max }^{+}\right\}} a_{j} \mathrm{e}^{\alpha_{j} t} \boldsymbol{f}_{\max }^{1,+} \boldsymbol{f}_{j}^{+}=0 ;
$$

however, this is a contradiction because $f^{+}$and $f_{\max }^{1,+}$ are nonnegative and

$$
\boldsymbol{f}_{\max }^{1,+} \mathrm{e}^{t \boldsymbol{G}^{+}} \boldsymbol{f}^{+}=\mathrm{e}^{\alpha_{\max } t} \boldsymbol{f}_{\max }^{1,+} \boldsymbol{f}^{+}>0 .
$$

Therefore, the vectors $f^{+}$and $f_{\max }^{+}$are not linearly independent.

Let the matrix $\boldsymbol{F}(y), y \in \mathbb{R}$, be defined by

$$
\boldsymbol{F}(y)= \begin{cases}\boldsymbol{J}_{1} \mathrm{e}^{y \boldsymbol{G}}=\mathrm{e}^{y \boldsymbol{G}} \boldsymbol{J}_{1}, & y>0, \\ \boldsymbol{J}_{2} \mathrm{e}^{y \boldsymbol{G}}=\mathrm{e}^{y \boldsymbol{G}} \boldsymbol{J}_{2}, & y<0 .\end{cases}
$$

Then we have the following result.

Lemma 3.7. For any $e, e^{\prime} \in E$,

$$
\begin{aligned}
& \mathrm{P}_{(e, \varphi)}\left(X_{H_{0}}=e^{\prime}, H_{0}<\infty\right)=[\boldsymbol{\Gamma} \boldsymbol{F}(-\varphi)]\left(e, e^{\prime}\right), \quad \varphi \neq 0, \\
& \mathrm{P}_{(e, 0)}\left(X_{H_{0}}=e^{\prime}, H_{0}<\infty\right)=\left[\boldsymbol{I}-\boldsymbol{\Gamma}_{2}\right]\left(e, e^{\prime}\right)=\left[\left(\begin{array}{cc}
\mathbf{0} & \boldsymbol{\Pi}^{-} \\
\boldsymbol{\Pi}^{+} & \mathbf{0}
\end{array}\right)\right]\left(e, e^{\prime}\right) .
\end{aligned}
$$

Proof. The lemma follows directly from the definition of the matrices $\boldsymbol{\Gamma}, \boldsymbol{\Gamma}_{2}$, and $\boldsymbol{F}(\varphi)$.

Let $g$ be the infinitesimal generator of the process $\left(X_{t}, \varphi_{t}\right)_{t \geq 0}$ and let $\mathscr{D}_{g}$ denote its domain. Let a function $f(e, \varphi)$ on $E \times \mathbb{R}$ be continuously differentiable in $\varphi$. Then $f \in \mathscr{D}_{q}$ and

$$
g f=\left(\boldsymbol{Q}+\boldsymbol{V} \frac{\partial}{\partial \varphi}\right) f
$$

where

$$
\begin{aligned}
\boldsymbol{Q f}(e, \varphi, t) & =\sum_{e^{\prime} \in E} \boldsymbol{Q}\left(e, e^{\prime}\right) f\left(e^{\prime}, \varphi\right), \\
\boldsymbol{V} \frac{\partial f}{\partial \varphi}(e, \varphi, t) & =\boldsymbol{V}(e, e) \frac{\partial f}{\partial \varphi}(e, \varphi) .
\end{aligned}
$$


Similarly, let $\mathcal{A}$ be the infinitesimal generator of the process $\left(X_{t}, \varphi_{t}, t\right)_{t \geq 0}$ and let $\mathcal{D}_{\mathcal{A}}$ denote its domain. Let a function $f(e, \varphi, t)$ on $E \times \mathbb{R} \times[0, \infty)$ be continuously differentiable in $\varphi$ and $t$. Then $f \in \mathscr{D}_{\mathcal{A}}$ and

$$
\mathcal{A} f=\left(\boldsymbol{Q}+\boldsymbol{V} \frac{\partial}{\partial \varphi}+\frac{\partial}{\partial t}\right) f .
$$

The behaviour of the process $\left(\varphi_{t}\right)_{t \geq 0}$ is determined by the matrices $\boldsymbol{Q}$ and $\boldsymbol{V}$. More precisely,

$$
\begin{aligned}
\left(\varphi_{t}\right)_{t \geq 0} \text { drifts to } \infty & \Longleftrightarrow \boldsymbol{\mu} \boldsymbol{V}>0 \\
& \Longleftrightarrow \boldsymbol{G}^{+} \text {is conservative and } \boldsymbol{G}^{-} \text {is not conservative, } \\
\left(\varphi_{t}\right)_{t \geq 0} \text { oscillates } & \Longleftrightarrow \boldsymbol{\mu} \boldsymbol{1}=0 \\
& \Longleftrightarrow \boldsymbol{G}^{+} \text {and } \boldsymbol{G}^{-} \text {are conservative, } \\
\left(\varphi_{t}\right)_{t \geq 0} \text { drifts to }-\infty & \Longleftrightarrow \boldsymbol{N} \mathbf{1}<0 \\
& \Longleftrightarrow \boldsymbol{G}^{-} \text {is conservative and } \boldsymbol{G}^{+} \text {is not conservative, }
\end{aligned}
$$

where $\boldsymbol{\mu}$ denotes the invariant measure of the process $\left(X_{t}\right)_{t \geq 0}$.

Let $\boldsymbol{f}_{1}=\boldsymbol{f}_{\max }$ and $\boldsymbol{g}_{1}=\boldsymbol{g}_{\text {min }}$ be the eigenvectors of $\boldsymbol{V}^{-1} \boldsymbol{Q}$ associated with the eigenvalues $\alpha_{\max }$ and $\beta_{\min }$, respectively. Then, in the positive-drift case $f_{\max }=\mathbf{1} \neq \boldsymbol{g}_{\min }$ and in the negative-drift case $\boldsymbol{g}_{\min }=\mathbf{1} \neq \boldsymbol{f}_{\mathrm{max}}$, and in both cases the basis $\mathcal{B}$ in the space of all vectors on $E$ is equal to $\left\{\boldsymbol{f}_{j}, j=1, \ldots, n ; \boldsymbol{g}_{k}, k=1, \ldots, m\right\}$. In the oscillating case, $\boldsymbol{f}_{\max }=\boldsymbol{g}_{\min }=\mathbf{1}$ and the equation $\boldsymbol{V}^{-1} \boldsymbol{Q x}=\mathbf{1}$ has a solution. If $\boldsymbol{r}$ is a solution then, by Jordan normal form theory, $\boldsymbol{r}$ is linearly independent of the vectors $\left\{\boldsymbol{f}_{j}, j=1, \ldots, n ; \boldsymbol{g}_{k}, k=1, \ldots, m\right\}$ and $\mathscr{B}=\left\{\mathbf{1}, \boldsymbol{r}, f_{j}, j=2, \ldots, n ; g_{k}, k=2, \ldots, m\right\}$ is a basis in the space of all vectors on $E$.

The following lemmas are concerned with the Perron-Frobenius eigenvalue of the matrix $\boldsymbol{Q}-\beta \boldsymbol{V}$. For any $\beta \in \mathbb{R}$, let $\alpha(\beta)$ be the Perron-Frobenius eigenvalue of the matrix $\boldsymbol{Q}-\beta \boldsymbol{V}$ and let $\boldsymbol{u}^{1}(\beta)$ and $\boldsymbol{u}^{\mathrm{r}}(\beta)$ be the associated left and right eigenvectors such that $\left\|\boldsymbol{u}^{1}(\beta)\right\|=\left\|\boldsymbol{u}^{\mathrm{r}}(\beta)\right\|=1$ in some norm in the space $\mathbb{R}^{|E|}$. A striking property of the eigenvalue $\alpha(\beta)$ is that it is a convex function of $\beta$.

Lemma 3.8. Let $\beta \in \mathbb{R}$ and let $\alpha(\beta)$ be the Perron-Frobenius eigenvalue of the matrix $\boldsymbol{Q}-\beta \boldsymbol{V}$. Then $\alpha(\beta)$ is a convex function of $\beta$ and is therefore continuous. It attains its global minimum and has two not necessarily distinct zeros, $\alpha_{\max } \leq 0$ and $\beta_{\min } \geq 0$.

Proof. Let $r(\boldsymbol{A})$ denote the Perron-Frobenius eigenvalue of an essentially nonnegative matrix $\boldsymbol{A}$. Since $\boldsymbol{Q}$ is essentially nonnegative it follows from Cohen (1981) that, for any $x, y \in \mathbb{R}$ and any $t, 0<t<1$,

$$
r((1-t)(\boldsymbol{Q}-x \boldsymbol{V})+t(\boldsymbol{Q}-y \boldsymbol{V})) \leq(1-t) r(\boldsymbol{Q}-x \boldsymbol{V})+\operatorname{tr}(\boldsymbol{Q}-y \boldsymbol{V}) .
$$

Hence, $\alpha(\beta)$ is a convex function and is therefore continuous.

For a $\beta$ with $|\beta|$ sufficiently large, some rows of $\boldsymbol{Q}-\beta \boldsymbol{V}$ are nonnegative, which implies that there exists no positive vector $\boldsymbol{f}$ such that $(\boldsymbol{Q}-\beta \boldsymbol{V}) \boldsymbol{f} \leq 0$. Hence, by the Perron-Frobenius theorem (see Theorem 2.6 of Seneta (1981)), $\alpha(\beta)>0$ for sufficiently large $|\beta|$.

Suppose that $\alpha(\beta)=0$. Then there exists a positive vector $\boldsymbol{f}$ such that $(\boldsymbol{Q}-\beta \boldsymbol{V}) \boldsymbol{f}=0$. Since, by Lemma 3.6(i), there exist exactly two (not necessarily distinct) eigenvalues of $\boldsymbol{V}^{-1} \boldsymbol{Q}$, $\alpha_{\max }$ and $\beta_{\min }$, whose associated eigenvectors are positive, it follows that $\alpha_{\max }$ and $\beta_{\min }$ are the only zeros of $\alpha(\beta)$.

Therefore, the function $\alpha(\beta)$ is continuous, for $|\beta|$ sufficiently large it is positive, and it has either one or two zeros. All of these together imply that $\alpha(\beta)$ attains its minimum. 
Lemma 3.9. Let $\alpha(\beta)$ be the Perron-Frobenius eigenvalue and let $\boldsymbol{u}^{1}(\beta)$ and $\boldsymbol{u}^{\mathrm{r}}(\beta)$ be the unit left and right Perron-Frobenius eigenvectors of the matrix $\boldsymbol{Q}-\beta \boldsymbol{V}$. Then $\alpha(\beta)$ is a differentiable function of $\beta$ and

$$
\frac{\mathrm{d} \alpha}{\mathrm{d} \beta}(\beta)=-\frac{\boldsymbol{u}^{\mathrm{l}}(\beta) \boldsymbol{V} \boldsymbol{u}^{\mathrm{r}}(\beta)}{\boldsymbol{u}^{\mathrm{l}}(\beta) \boldsymbol{u}^{\mathrm{r}}(\beta)} .
$$

In addition, there is a unique $\beta_{0} \in\left(\alpha_{\max }, \beta_{\min }\right)$ such that $(\mathrm{d} \alpha / \mathrm{d} \beta)\left(\beta_{0}\right)=0, \alpha_{0} \equiv \alpha\left(\beta_{0}\right)$ is the global minimum of the function $\alpha(\beta)$, and

$$
\frac{\mathrm{d} \alpha}{\mathrm{d} \beta}(\beta) \begin{cases}<0 & \text { if } \beta<\beta_{0}, \\ =0 & \text { if } \beta=\beta_{0}, \\ >0 & \text { if } \beta>\beta_{0} .\end{cases}
$$

Proof. By multiplying the equality

$$
\begin{aligned}
& (\boldsymbol{Q}-\beta \boldsymbol{V}) \boldsymbol{u}^{\mathrm{r}}(\beta+h)-h \boldsymbol{V} \boldsymbol{u}^{\mathrm{r}}(\beta+h) \\
& \quad=(\alpha(\beta+h)-\alpha(\beta)) \boldsymbol{u}^{\mathrm{r}}(\beta+h)+\alpha(\beta) \boldsymbol{u}^{\mathrm{r}}(\beta+h)
\end{aligned}
$$

by $\boldsymbol{u}^{1}(\beta) / h$ (on the left) and letting $h \rightarrow 0$, we find that $\alpha(\beta)$ is a differentiable function of $\beta$. By Lemma 3.8, it is also convex and attains its minimum. Hence, there exists a unique $\beta_{0}$ such that $\alpha\left(\beta_{0}\right)$ is the global minimum of $\alpha(\beta)$ and $(\mathrm{d} \alpha / \mathrm{d} \beta)\left(\beta_{0}\right)=0$. Also by Lemma 3.8, $\alpha(\beta)$ has two zeros, $\alpha_{\max } \leq 0$ and $\beta_{\min } \geq 0$. Hence, $\beta_{0} \in\left(\alpha_{\max }, \beta_{\min }\right)$ when $\alpha_{\max } \neq \beta_{\min }$ and $\beta_{0}=\alpha_{\max }=\beta_{\min }$ when $\alpha_{\max }=\beta_{\min }$.

It remains to show that $\alpha(\beta)$ is strictly monotone on $\left(-\infty, \beta_{0}\right]$ and $\left[\beta_{0}, \infty\right)$. Let $\alpha(\beta)$ be the Perron-Frobenius eigenvalue of the matrix $\boldsymbol{Q}-\beta \boldsymbol{V}$, and first suppose that $\beta_{0}=0$. Then $\alpha\left(\beta_{0}\right)=0$ and, therefore, $\alpha(\beta) \geq 0$. By Lemma 3.6(i), for $\alpha>0$ the only positive eigenvectors of $\boldsymbol{V}^{-1}(\boldsymbol{Q}-\alpha \boldsymbol{I})$ are $\boldsymbol{f}_{\max }(\alpha)$ and $\boldsymbol{g}_{\min }(\alpha)$, which are associated with the eigenvalues $\alpha_{\max }(\alpha)$ and $\beta_{\min }(\alpha)$, respectively. Hence, for a fixed $\alpha \geq \alpha_{0}$, there exist only two values of $\beta$, namely $\alpha_{\max }(\alpha)$ and $\beta_{\min }(\alpha)$, such that $\alpha$ is the Perron-Frobenius eigenvalue of $\boldsymbol{Q}-\beta \boldsymbol{V}$. Since $\alpha_{\max }(\alpha) \leq 0$ and $\beta_{\min }(\alpha) \geq 0$, it follows that $\alpha(\beta)$ is strictly monotone on both intervals $(-\infty, 0]$ and $[0, \infty)$.

Now let $\beta_{0} \neq 0$ and $\boldsymbol{Q}_{0}=\boldsymbol{Q}-\beta_{0} \boldsymbol{V}-\alpha_{0} \boldsymbol{I}$. The matrix $\boldsymbol{Q}_{0}$ is essentially nonnegative and (by Lemma 3.1) irreducible; so is the matrix $\boldsymbol{Q}_{0}-\beta \boldsymbol{V}$, for any $\beta \in \mathbb{R}$. Let $\alpha_{0}(\beta)$ be the Perron-Frobenius eigenvalue of $\boldsymbol{Q}_{0}-\beta \boldsymbol{V}$. Then $\alpha_{0}(\beta)=\alpha\left(\beta+\beta_{0}\right)-\alpha_{0}$. Since $\alpha(\beta)$ attains its global minimum at $\beta=\beta_{0}$, it follows that $\alpha_{0}(\beta)$ attains its global minimum, i.e. 0 , at $\beta=0$. Therefore, by the argument for the $\beta_{0}=0$ case, $\alpha_{0}(\beta)$ is strictly monotone on $(-\infty, 0]$ and $[0, \infty)$, which implies that $\alpha(\beta)$ is strictly monotone on $\left(-\infty, \beta_{0}\right]$ and $\left[\beta_{0}, \infty\right)$.

The sign of the unique argument, $\beta_{0}$, of the global minimum of the function $\alpha(\beta)$, whose existence we have just proved, is found to depend on the behaviour of the process $\left(\varphi_{t}\right)_{t \geq 0}$. This is the result of the next lemma.

Lemma 3.10. (i) In the positive-drift case, $\beta_{0}>0$ and $\alpha_{0}<0$.

(ii) In the oscillating case, $\beta_{0}=0$ and $\alpha_{0}=0$.

(iii) In the negative-drift case, $\beta_{0}<0$ and $\alpha_{0}<0$. 
Proof. In the drift cases $\alpha_{\max } \neq \beta_{\min }$ and, therefore, by Lemma 3.9, $\beta_{0} \in\left(\alpha_{\max }, \beta_{\min }\right)$. In the positive-drift case, by (3.6), $\alpha_{\max }=0$ and $\beta_{\min }>0$ and, therefore, $\beta_{0}>0$. In the negative-drift case, by (3.6), $\beta_{\min }=0$ and $\alpha_{\max }<0$ and, therefore, $\beta_{0}<0$. Since, in both cases, the function $\alpha(\beta)$ has two distinct zeros, its global minimum $\alpha_{0}$ is negative.

Finally, in the oscillating case, by (3.6), $\alpha_{\max }=\beta_{\min }=0$ and, therefore, $\beta_{0}=0$. Thus, the function $\alpha(\beta)$ has exactly one zero at $\beta=0$ and, since by Lemma 3.8 it attains a global minimum, it follows that $\alpha(\beta)$ attains its global minimum at $\beta_{0}=0$ and that $\alpha_{0}=\alpha\left(\beta_{0}\right)=0$.

Lemma 3.11. The matrix $Q^{0}$ given by (2.1) is a conservative irreducible $Q$-matrix. In addition, if $\boldsymbol{\mu}^{0}$ is a vector on $E$ such that $\boldsymbol{\mu}^{0} \boldsymbol{Q}^{0}=\mathbf{0}$, then $\boldsymbol{\mu}^{0} \boldsymbol{V} \mathbf{1}=0$.

Proof. Since the matrices $\boldsymbol{I}$ and $\boldsymbol{V}$ are diagonal and the vector $\boldsymbol{g}_{0}$ is positive, the matrix $\boldsymbol{Q}^{0}$ is essentially nonnegative. In addition, $\boldsymbol{Q}^{0} \mathbf{1}=\mathbf{0}$.

By Lemma 3.1, the matrix $\boldsymbol{Q}-\alpha_{0} \boldsymbol{I}-\beta_{0} \boldsymbol{V}$ is irreducible, which implies that the matrix $\mathrm{e}^{t\left(\boldsymbol{Q}-\alpha_{0} \boldsymbol{I}-\beta_{0} \boldsymbol{V}\right)}$ is positive for all $t>0$. Since the vector $\boldsymbol{g}_{0}$ is positive, it follows from the definition of $\boldsymbol{Q}^{0}$ that $\mathrm{e}^{t} \boldsymbol{Q}^{0}$ is positive for all $t>0$ and that the matrix $\boldsymbol{Q}^{0}$ is irreducible.

Let $\boldsymbol{g}_{0}^{1}$ be the left Perron-Frobenius eigenvector of the matrix $\boldsymbol{Q}-\beta_{0} \boldsymbol{V}$, and let $\boldsymbol{\mu}^{0}$ be a vector on $E$ with entries $\mu^{0}(e)=g_{0}^{1}(e) g_{0}(e), e \in E$. Then $\boldsymbol{\mu}^{0} \boldsymbol{Q}^{0}=\mathbf{0}$ and, by Lemmas 3.9 and 3.10, $\boldsymbol{\mu}^{0} \boldsymbol{V} \mathbf{1}=0$. Since any vector $\boldsymbol{v}$ that satisfies $\boldsymbol{v} \boldsymbol{Q}^{0}=\mathbf{0}$ is a constant multiple of $\boldsymbol{\mu}^{0}$, the proof of the lemma is complete.

We recall the matrix $\boldsymbol{G}_{0}=\operatorname{diag}\left(\boldsymbol{g}_{0}\right)$. Since the vector $\boldsymbol{g}_{0}$ is positive, the matrix $\boldsymbol{G}_{0}$ is invertible.

Lemma 3.12. For $\alpha>0$, let

$$
\boldsymbol{V}^{-1}(\boldsymbol{Q}-\alpha \boldsymbol{I}) \boldsymbol{\Gamma}_{\alpha}=\boldsymbol{\Gamma}_{\alpha} \boldsymbol{G}_{\alpha} \quad \text { and } \quad \boldsymbol{V}^{-1}\left(\boldsymbol{Q}^{0}-\alpha \boldsymbol{I}\right) \boldsymbol{\Gamma}_{\alpha}^{0}=\boldsymbol{\Gamma}_{\alpha}^{0} \boldsymbol{G}_{\alpha}^{0}
$$

be the Wiener-Hopf factorizations of $\boldsymbol{V}^{-1}(\boldsymbol{Q}-\alpha \boldsymbol{I})$ and $\boldsymbol{V}^{-1}\left(\boldsymbol{Q}^{0}-\alpha \boldsymbol{I}\right)$, respectively. Then

$$
\boldsymbol{G}_{\alpha-\alpha_{0}}^{0}=\boldsymbol{G}_{0}^{-1}\left(\boldsymbol{G}_{\alpha}-\beta_{0} \boldsymbol{I}\right) \boldsymbol{G}_{0} \quad \text { and } \quad \boldsymbol{\Gamma}_{\alpha-\alpha_{0}}^{0}=\boldsymbol{G}_{0}^{-1} \boldsymbol{\Gamma}_{\alpha} \boldsymbol{G}_{0}, \quad \alpha>0 .
$$

Proof. By the definition of $\boldsymbol{Q}^{0}$ and by the Wiener-Hopf factorization of $\boldsymbol{V}^{-1}(\boldsymbol{Q}-\alpha \boldsymbol{I})$, $\alpha>0$, given in Lemma 3.2, we have

$$
\boldsymbol{V}^{-1}\left(\boldsymbol{Q}^{0}-\left(\alpha-\alpha_{0}\right) \boldsymbol{I}\right)=\boldsymbol{G}_{0}^{-1} \boldsymbol{\Gamma}_{\alpha} \boldsymbol{G}_{0}\left(\boldsymbol{G}_{0}^{-1}\left(\boldsymbol{G}_{\alpha}-\beta_{0} \boldsymbol{I}\right) \boldsymbol{G}_{0}\right) \boldsymbol{G}_{0}^{-1} \boldsymbol{\Gamma}_{\alpha}^{-1} \boldsymbol{G}_{0}
$$

Let $\boldsymbol{G}_{0}^{+}$and $\boldsymbol{G}_{0}^{-}$be the restrictions of $\boldsymbol{G}_{0}$ to $E^{+} \times E^{+}$and, respectively, $E^{-} \times E^{-}$. Then

$$
\boldsymbol{G}_{0}^{-1}\left(\boldsymbol{G}_{\alpha}-\beta_{0} \boldsymbol{I}\right) \boldsymbol{G}_{0}=\left(\begin{array}{cc}
\left(\boldsymbol{G}_{0}^{+}\right)^{-1}\left(\boldsymbol{G}_{\alpha}^{+}-\beta_{0} \boldsymbol{I}\right) \boldsymbol{G}_{0}^{+} & \mathbf{0} \\
\mathbf{0} & -\left(\boldsymbol{G}_{0}^{-}\right)^{-1}\left(\boldsymbol{G}_{\alpha}^{-}+\beta_{0} \boldsymbol{I}\right) \boldsymbol{G}_{0}^{-}
\end{array}\right) .
$$

Suppose that $\left(\boldsymbol{G}_{0}^{+}\right)^{-1}\left(\boldsymbol{G}_{\alpha}^{+}-\beta_{0} \boldsymbol{I}\right) \boldsymbol{G}_{0}^{+}$and $\left(\boldsymbol{G}_{0}^{-}\right)^{-1}\left(\boldsymbol{G}_{\alpha}^{-}+\beta_{0} \boldsymbol{I}\right) \boldsymbol{G}_{0}^{-}$are $Q$-matrices. Then, by Lemma 3.2, (3.7) is the Wiener-Hopf factorization of $\boldsymbol{V}^{-1}\left(\boldsymbol{Q}^{0}-\left(\alpha-\alpha_{0}\right) \boldsymbol{I}\right)$ for $\alpha>0$, and, by the uniqueness of the Wiener-Hopf factorization,

$$
\boldsymbol{G}_{\alpha-\alpha_{0}}^{0}=\boldsymbol{G}_{0}^{-1}\left(\boldsymbol{G}_{\alpha}-\beta_{0} \boldsymbol{I}\right) \boldsymbol{G}_{0} \quad \text { and } \quad \boldsymbol{\Gamma}_{\alpha-\alpha_{0}}^{0}=\boldsymbol{G}_{0}^{-1} \boldsymbol{\Gamma}_{\alpha} \boldsymbol{G}_{0}, \quad \alpha>0 .
$$

Therefore, all we have to prove is that $\left(\boldsymbol{G}_{0}^{+}\right)^{-1}\left(\boldsymbol{G}_{\alpha}^{+}-\beta_{0} \boldsymbol{I}\right) \boldsymbol{G}_{0}^{+}$and $\left(\boldsymbol{G}_{0}^{-}\right)^{-1}\left(\boldsymbol{G}_{\alpha}^{-}+\beta_{0} \boldsymbol{I}\right) \boldsymbol{G}_{0}^{-}$are $Q$-matrices.

Let the function $\boldsymbol{h}$ be defined by $\boldsymbol{h}(e, \varphi, t)=\mathrm{e}^{-\alpha_{0} t} \mathrm{e}^{-\beta_{0} \varphi} \boldsymbol{g}_{0}(e)$. Then $\boldsymbol{h}$ is continuously differentiable in $\varphi$ and $t$ and, by (3.5), it is in the domain of the infinitesimal generator $\mathcal{A}$ of the 
process $\left(X_{t}, \varphi_{t}, t\right)_{t \geq 0}$, with $\mathcal{A} \boldsymbol{h}=\mathbf{0}$. It follows that the process $\left(\boldsymbol{h}\left(X_{t \wedge H_{y}}, \varphi_{t \wedge H_{y}}, t \wedge H_{y}\right)\right)_{t \geq 0}$ is a positive martingale. By Fatou's lemma,

$$
\mathrm{E}_{(e, \varphi)}\left(\mathrm{e}^{-\alpha_{0} H_{y}} \mathrm{e}^{-\beta_{0} \varphi_{H_{y}}} \boldsymbol{g}_{0}\left(X_{H_{y}}\right)\right) \leq \mathrm{e}^{-\beta_{0} \varphi} \boldsymbol{g}_{0}(e),
$$

and, because $\boldsymbol{g}_{0}$ is positive,

$$
\mathrm{E}_{(e, \varphi)}\left(\mathrm{e}^{-\alpha H_{y}} \boldsymbol{g}_{0}\left(X_{H_{y}}\right)\right) \leq \mathrm{E}_{(e, \varphi)}\left(\mathrm{e}^{-\alpha_{0} H_{y}} \boldsymbol{g}_{0}\left(X_{H_{y}}\right)\right) \leq \mathrm{e}^{-\beta_{0}(\varphi-y)} \boldsymbol{g}_{0}(e)
$$

for $\alpha>\alpha_{0}$.

By Lemma 3.3, for $\varphi=0$ and $y>0$, we have

$$
\mathrm{e}^{-\beta_{0} y} \boldsymbol{g}_{0}(e) \geq \mathrm{E}_{(e, 0)}\left(\mathrm{e}^{-\alpha H_{y}} \boldsymbol{g}_{0}\left(X_{H_{y}}\right)\right)=\left(\begin{array}{c}
\mathrm{e}^{y \boldsymbol{G}_{\alpha}^{+}} \boldsymbol{g}_{0}^{+} \\
\boldsymbol{\Pi}_{\alpha}^{+} \mathrm{e}^{y \boldsymbol{G}_{\alpha}^{+}} \boldsymbol{g}_{0}^{+}
\end{array}\right),
$$

which implies that $\mathrm{e}^{y\left(\boldsymbol{G}_{\alpha}^{+}-\beta_{0} \boldsymbol{I}\right)} \boldsymbol{g}_{0}^{+} \leq \boldsymbol{g}_{0}^{+}$, componentwise. Hence, because

$$
\lim _{y \rightarrow 0} \frac{\mathrm{e}^{y\left(\boldsymbol{G}_{\alpha}^{+}-\beta_{0} \boldsymbol{I}\right)} \boldsymbol{g}_{0}^{+}-\boldsymbol{g}_{0}^{+}}{y}=\left(\boldsymbol{G}_{\alpha}^{+}-\beta_{0} \boldsymbol{I}\right) \boldsymbol{g}_{0}^{+},
$$

we have $\left(\boldsymbol{G}_{\alpha}^{+}-\beta_{0} \boldsymbol{I}\right) g_{0}^{+} \leq 0$ and, therefore, because $\left(\boldsymbol{G}_{0}^{+}\right)^{-1}$ is positive,

$$
\left(\boldsymbol{G}_{0}^{+}\right)^{-1}\left(\boldsymbol{G}_{\alpha}^{+}-\beta_{0} \boldsymbol{I}\right) \boldsymbol{G}_{0}^{+} \mathbf{1}^{+}=\left(\boldsymbol{G}_{0}^{+}\right)^{-1}\left(\boldsymbol{G}_{\alpha}^{+}-\beta_{0} \boldsymbol{I}\right) \boldsymbol{g}_{0}^{+} \leq 0
$$

and $\left(\boldsymbol{G}_{0}^{+}\right)^{-1}\left(\boldsymbol{G}_{\alpha}^{+}-\beta_{0} \boldsymbol{I}\right) \boldsymbol{G}_{0}^{+}$is a $Q$-matrix. It can be proved that $\left(\boldsymbol{G}_{0}^{-}\right)^{-1}\left(\boldsymbol{G}_{\alpha}-\beta_{0} \boldsymbol{I}\right) \boldsymbol{G}_{0}^{-}$is a $Q$-matrix in the same way.

Theorem 3.1. For $\alpha \geq 0$, let $\alpha_{\max }(\alpha)$ and $\beta_{\min }(\alpha)$ be the eigenvalues of the matrix $\boldsymbol{V}^{-1}(\boldsymbol{Q}-\alpha \boldsymbol{I})$ with maximal negative and minimal positive real parts, respectively, and let $f_{\max }(\alpha)$ and $g_{\min }(\alpha)$ be their associated eigenvectors, respectively.

Then, in the oscillating case, there exists an $\varepsilon>0$ such that, for $0<\alpha<\varepsilon$, and some constants $d_{n}, n=2,3, \ldots$, and $c>0$, we have

$$
\begin{aligned}
& \alpha_{\max }(\alpha)=-\frac{1}{\sqrt{-\boldsymbol{\mu V} \boldsymbol{r}}} \alpha^{1 / 2}+d_{2} \alpha+d_{3} \alpha^{3 / 2}+\cdots=-\frac{1}{\sqrt{-\boldsymbol{\mu} \boldsymbol{V} \boldsymbol{r}}} \alpha^{1 / 2}+\Theta_{\max }\left(\alpha^{1 / 2}\right), \\
& \beta_{\min }(\alpha)=\frac{1}{\sqrt{-\boldsymbol{\mu} \boldsymbol{V} \boldsymbol{r}}} \alpha^{1 / 2}+d_{2} \alpha-d_{3} \alpha^{3 / 2}+\cdots=\frac{1}{\sqrt{-\boldsymbol{\mu} \boldsymbol{V} \boldsymbol{r}}} \alpha^{1 / 2}+\Theta_{\min }\left(\alpha^{1 / 2}\right),
\end{aligned}
$$

where $\left|\Theta_{\max }\left(\alpha^{1 / 2}\right)\right|<c \alpha$ and $\left|\Theta_{\min }\left(\alpha^{1 / 2}\right)\right|<c \alpha$.

For suitable vectors $\boldsymbol{v}_{2}, \boldsymbol{w}_{2}, \boldsymbol{\Xi}_{\max }\left(\alpha^{1 / 2}\right)$, and $\boldsymbol{\Xi}_{\min }\left(\alpha^{1 / 2}\right)$, the vectors $\boldsymbol{f}_{\max }(\alpha)$ and $\boldsymbol{g}_{\min }(\alpha)$ can be chosen to be

$$
\begin{aligned}
& \boldsymbol{f}_{\max }(\alpha)=\mathbf{1}-\frac{1}{\sqrt{-\boldsymbol{\mu} \boldsymbol{V} \boldsymbol{r}}} \alpha^{1 / 2} \boldsymbol{r}+\alpha \boldsymbol{v}_{2}+\cdots=\mathbf{1}-\frac{1}{\sqrt{-\boldsymbol{\mu V \boldsymbol { r }}}} \alpha^{1 / 2} \boldsymbol{r}+\boldsymbol{\Xi}_{\max }\left(\alpha^{1 / 2}\right), \\
& \boldsymbol{g}_{\min }(\alpha)=\mathbf{1}+\frac{1}{\sqrt{-\boldsymbol{\mu} \boldsymbol{V} \boldsymbol{r}}} \alpha^{1 / 2} \boldsymbol{r}+\alpha \boldsymbol{w}_{2}+\cdots=\mathbf{1}+\frac{1}{\sqrt{-\boldsymbol{\mu} \boldsymbol{V} \boldsymbol{r}}} \alpha^{1 / 2} \boldsymbol{r}+\boldsymbol{\Xi}_{\min }\left(\alpha^{1 / 2}\right),
\end{aligned}
$$

where $\boldsymbol{V}^{-1} \boldsymbol{Q} \boldsymbol{r}=\mathbf{1}$ and $\left\|\boldsymbol{\Xi}_{\max }\left(\alpha^{1 / 2}\right)\right\|<\alpha v$ and $\left\|\boldsymbol{\Xi}_{\min }\left(\alpha^{1 / 2}\right)\right\|<\alpha w$ for some positive scalars $v$ and $w$. 
In the negative-drift case, there exists an $\varepsilon>0$ such that, for $0<\alpha<\varepsilon$, and some constants $a_{n}$ and $b_{n}, n \in \mathbb{N}$, we have

$$
\alpha_{\max }(\alpha)=\alpha_{\max }+a_{1} \alpha+a_{2} \alpha^{2}+\cdots \quad \text { and } \beta_{\min }(\alpha)=b_{1} \alpha+b_{2} \alpha^{2}+b_{3} \alpha^{3}+\cdots,
$$

and the vectors $\boldsymbol{f}_{\max }(\alpha)$ and $\boldsymbol{g}_{\min }(\alpha)$ can be chosen to be

$$
\boldsymbol{f}_{\max }(\alpha)=\boldsymbol{f}_{\max }+\alpha \boldsymbol{v}_{1}+\alpha^{2} \boldsymbol{v}_{2}+\cdots \quad \text { and } \boldsymbol{g}_{\min }(\alpha)=\mathbf{1}+\alpha \boldsymbol{w}_{1}+\alpha^{2} \boldsymbol{w}_{2}+\cdots,
$$

where $\boldsymbol{v}_{n}$ and $\boldsymbol{w}_{n}, n \in \mathbb{N}$, are constant vectors.

An analogous result holds in the positive-drift case.

Proof. The eigenvalues of $\boldsymbol{V}^{-1}(\boldsymbol{Q}-\alpha \boldsymbol{I})$ converge to the eigenvalues of $\boldsymbol{V}^{-1} \boldsymbol{Q}$ as $\alpha \rightarrow 0$. Thus, $\alpha_{\max }(\alpha) \rightarrow \alpha_{\max }$ and $\beta_{\min }(\alpha) \rightarrow \beta_{\min }$ as $\alpha \rightarrow 0$.

In the drift cases, by (3.6), $\alpha_{\max } \neq \beta_{\min }$. Hence, $\alpha_{\max }$ and $\beta_{\min }$ are simple eigenvalues of $\boldsymbol{V}^{-1} \boldsymbol{Q}$, which implies, for a sufficiently small $\alpha>0$, that $\alpha_{\max }(\alpha)$ and $\beta_{\min }(\alpha)$, and also $f_{\max }(\alpha)$ and $\boldsymbol{g}_{\min }(\alpha)$, can be represented by convergent power series (see Wilkinson (1965)). In addition, in the positive-drift case $\alpha_{\max }=0$ and $\boldsymbol{f}_{\max }=\mathbf{1}$, and in the negative-drift case $\beta_{\min }=0$ and $\boldsymbol{g}_{\min }=\mathbf{1}$. This proves the theorem for the drift cases.

In the oscillating case, by (3.6), 0 is an eigenvalue of the matrix $\boldsymbol{V}^{-1} \boldsymbol{Q}$ with algebraic multiplicity two. Hence, there exists an $\varepsilon>0$ such that, for $0<|\alpha|<\varepsilon$, there exist two eigenvalues of $\boldsymbol{V}^{-1}(\boldsymbol{Q}-\alpha \boldsymbol{I})$, namely $\alpha_{\max }(\alpha)$ and $\beta_{\min }(\alpha)$, that converge to 0 as $\alpha \rightarrow 0$. In addition, either

$$
\begin{aligned}
& \alpha_{\max }(\alpha)=a_{1} \alpha+a_{2} \alpha^{2}+a_{3} \alpha^{3}+\cdots, \\
& \beta_{\min }(\alpha)=b_{1} \alpha+b_{2} \alpha^{2}+b_{3} \alpha^{3}+\cdots,
\end{aligned}
$$

for some constants $a_{k}$ and $b_{k}, k \in \mathbb{N}$, or

$$
\begin{aligned}
& \alpha_{\max }(\alpha)=d_{1} \alpha^{1 / 2}+d_{2} \alpha+d_{3} \alpha^{3 / 2}+\cdots, \\
& \beta_{\min }(\alpha)=-d_{1} \alpha^{1 / 2}+d_{2} \alpha-d_{3} \alpha^{3 / 2}+\cdots,
\end{aligned}
$$

for some constants $d_{k}, k \in \mathbb{N}$. We shall show that (3.8) is not possible.

For any $\alpha>0$,

$$
\left(\boldsymbol{Q}-\alpha_{\max }(\alpha) \boldsymbol{V}\right) \boldsymbol{f}_{\max }(\alpha)=\alpha \boldsymbol{f}_{\max }(\alpha) .
$$

Since, by Lemma 3.1, the matrix $\boldsymbol{Q}-\alpha_{\max } \boldsymbol{V}$ is irreducible and essentially nonnegative and the vector $f_{\max }(\alpha)$ is positive, it follows that $\alpha$ is the Perron-Frobenius eigenvalue of $\boldsymbol{Q}-\alpha_{\max }(\alpha) \boldsymbol{V}$. Similarly, $\alpha$ is the Perron-Frobenius eigenvalue of $\boldsymbol{Q}-\beta_{\min }(\alpha) \boldsymbol{V}$.

Let $\beta \in \mathbb{R}$ and consider the matrix $\boldsymbol{Q}-\beta \boldsymbol{V}$ and its Perron-Frobenius eigenvalue $\alpha(\beta)$ and eigenvector $\boldsymbol{u}(\beta)$. The eigenvalue $\alpha(\beta)$ is simple and converges to a simple eigenvalue of the matrix $Q$ as $\beta \rightarrow 0$. Thus, for $|\beta|<\delta$,

$$
\begin{aligned}
& \alpha(\beta)=c_{0}+c_{1} \beta+c_{2} \beta^{2}+\cdots, \\
& \boldsymbol{u}(\beta)=\mathbf{1}+\beta \boldsymbol{v}_{1}+\beta^{2} \boldsymbol{v}_{2}+\cdots,
\end{aligned}
$$

for some constants $c_{k}, k \in \mathbb{N} \cup\{0\}$, and some vectors $\boldsymbol{v}_{k}, k \in \mathbb{N}$, on $E$.

Suppose that the process $\left(\varphi_{t}\right)_{t \geq 0}$ oscillates. By Lemmas 3.9 and 3.10, the eigenvalue $\alpha(\beta)$ attains its global minimum, 0 , at $\beta=0$. Hence, $\alpha(0)=(\mathrm{d} \alpha / \mathrm{d} \beta)(0)=0$, which gives $c_{0}=c_{1}=0$ and, therefore,

$$
\alpha(\beta)=c_{2} \beta^{2}+c_{3} \beta^{3}+c_{4} \beta^{4}+\cdots
$$


By substituting $\alpha(\beta)$ and $\boldsymbol{u}(\beta)$ into the equation

$$
(\boldsymbol{Q}-\beta \boldsymbol{V}) \boldsymbol{u}(\beta)=\alpha(\beta) \boldsymbol{u}(\beta),
$$

and by equating the coefficients of $\beta$ and $\beta^{2}$ on each side of the resulting expansion, we obtain

$$
\boldsymbol{V}^{-1} \boldsymbol{Q} \boldsymbol{v}_{1}=1, \quad \boldsymbol{Q} \boldsymbol{v}_{2}-\boldsymbol{V} \boldsymbol{v}_{1}=c_{2} \mathbf{1}
$$

It follows that $c_{2} \neq 0$. (If $c_{2}=0$ then $\boldsymbol{V}^{-1} \boldsymbol{Q} \boldsymbol{v}_{2}=\boldsymbol{v}_{1}$, which, by Jordan matrix theory, is not possible since 0 is the eigenvalue of $\boldsymbol{V}^{-1} \boldsymbol{Q}$ with algebraic multiplicity two.)

Suppose that (3.8) holds. Then it follows from (3.8) and (3.10) that, for $|\alpha|<\varepsilon$,

$$
\begin{aligned}
\alpha=\alpha\left(\alpha_{\max }\right) & =c_{2} \alpha_{\max }^{2}(\alpha)+c_{3} \alpha_{\max }^{3}(\alpha)+\cdots \\
& =c_{2}\left(a_{1} \alpha+a_{2} \alpha^{2}+\cdots\right)^{2}+c_{3}\left(a_{1} \alpha+a_{2} \alpha^{2}+\cdots\right)^{3}+\cdots \\
& =c_{2} a_{1}^{2} \alpha^{2}+\text { const. } \alpha^{3}+\cdots
\end{aligned}
$$

which is not possible for every $|\alpha|<\varepsilon$. Hence, (3.8) is not true and, thus, (3.9) holds.

Substituting $\alpha_{\max }(\alpha)$ and $\beta_{\min }(\alpha)$ from (3.9) into (3.12) gives $d_{1}^{2}=1 / c_{2}$. By Lemmas 3.9 and 3.10, $\alpha(0)=0$ is the minimum of the function $\alpha(\beta)$, which implies that $\alpha(\beta)>0$ for all $\beta \in \mathbb{R}$, and, by (3.12), that $c_{2}>0$. By multiplying the second equality in (3.13) by $\mu$ on the left, we obtain $c_{2}=-\boldsymbol{\mu} \boldsymbol{V} \boldsymbol{v}_{1} / \boldsymbol{\mu} \mathbf{1}=-\boldsymbol{\mu} \boldsymbol{V} \boldsymbol{v}_{1}$ (because $\boldsymbol{\mu} \mathbf{1}=1$ ). Therefore, the statement in the theorem follows from (3.9) and (3.11).

\section{The oscillating case: proof of Theorem 2.1}

We start by considering $\lim _{T \rightarrow \infty} \mathrm{P}_{(e, \varphi)}^{(T)}(A)$ for $A \in \mathcal{F}_{t}$. By Lemma 3.5(viii), the events $\left\{H_{0}>T\right\}, T>0$, are of positive probability. Thus, for $0<t<T$ and $A \in \mathcal{F}_{t}$,

$$
\mathrm{P}_{(e, \varphi)}^{(T)}(A)=\mathrm{P}_{(e, \varphi)}\left(A \mid H_{0}>T\right)=\frac{\mathrm{E}_{(e, \varphi)}\left(1(A) \mathrm{P}_{\left(X_{t}, \varphi_{t}\right)}\left(H_{0}>T-t\right) 1\left(H_{0}>t\right)\right)}{\mathrm{P}_{(e, \varphi)}\left(H_{0}>T\right)}
$$

We first show that

$$
\lim _{T \rightarrow \infty} \frac{\mathrm{P}_{\left(e^{\prime}, \varphi^{\prime}\right)}\left(H_{0}>T-t\right)}{\mathrm{P}_{(e, \varphi)}\left(H_{0}>T\right)}
$$

exists by looking at the asymptotic behaviour of the function $t \mapsto \mathrm{P}_{(e, \varphi)}\left(H_{0}>t\right)$.

In the oscillating case, by (3.6) and Lemma 3.5(iv), 0 is an eigenvalue of $\boldsymbol{V}^{-1} \boldsymbol{Q}$ with algebraic multiplicity two and geometric multiplicity one. Therefore, there exists a vector $\boldsymbol{r}$ such that $\boldsymbol{V}^{-1} \boldsymbol{Q} \boldsymbol{r}=\mathbf{1}$. Since the choice of such vector is not relevant in the present work, we shall always refer to it as if it were fixed.

Recall that $\boldsymbol{\mu}$ is the invariant measure of the process $\left(X_{t}\right)_{t \geq 0}$.

Lemma 4.1. For any $(e, \varphi) \in E_{0}^{+}$,

$$
\begin{aligned}
& \mathrm{P}_{(e, \varphi)}\left(H_{0}>t\right) \sim \frac{1}{\pi} \frac{1}{\sqrt{-\boldsymbol{\mu} \boldsymbol{V} \boldsymbol{r}}} t^{-1 / 2}\left[-\mathrm{e}^{-\varphi \boldsymbol{V}^{-1} \boldsymbol{Q}} \boldsymbol{J}_{1} \boldsymbol{\Gamma}_{2} \boldsymbol{r}\right](e), \quad t \rightarrow \infty, \\
& h_{\boldsymbol{r}}(e, \varphi):=-\left[\mathrm{e}^{-\varphi \boldsymbol{V}^{-1} \boldsymbol{Q}} \boldsymbol{J}_{1} \boldsymbol{\Gamma}_{2} \boldsymbol{r}\right](e)>0 .
\end{aligned}
$$


Proof. (i) The statement is proved by applying Tauberian theorems to the Laplace transform, $1-\mathrm{E}_{(e, \varphi)}\left(\mathrm{e}^{-\alpha H_{0}}\right) / \alpha$, of $\mathrm{P}_{(e, \varphi)}\left(H_{0}>t\right)$. By Lemmas 3.2 and 3.3, for $\alpha>0$ and $(e, \varphi) \in E_{0}^{+}$, we have

$$
\frac{1-\mathrm{E}_{(e, \varphi)}\left(\mathrm{e}^{-\alpha H_{0}}\right)}{\alpha}=\left[\mathrm{e}^{-\varphi \boldsymbol{V}^{-1} \boldsymbol{Q}} \frac{\mathbf{1}-\boldsymbol{\Gamma}_{\alpha} \boldsymbol{J}_{2} \mathbf{1}}{\alpha}\right](e)-\left[\frac{\mathrm{e}^{-\varphi \boldsymbol{V}^{-1}(\boldsymbol{Q}-\alpha \boldsymbol{I})}-\mathrm{e}^{-\varphi \boldsymbol{V}^{-1} \boldsymbol{Q}}}{\alpha} \boldsymbol{\Gamma}_{\alpha} \boldsymbol{J}_{2} \mathbf{1}\right]_{(}(e) .
$$

Let $\beta_{\min }(\alpha)$ be the eigenvalue of $\boldsymbol{V}^{-1}(\boldsymbol{Q}-\alpha \boldsymbol{I})$ with minimal positive real part and let $\boldsymbol{g}_{\min }(\alpha)$ be its associated eigenvector. Then, by (3.2), $\boldsymbol{\Pi}_{\alpha}^{-} \boldsymbol{g}_{\min }^{-}(\alpha)=\boldsymbol{g}_{\min }^{+}(\alpha)$ and, by substituting $\boldsymbol{g}_{\min }(\alpha)$ from Theorem 3.1, we obtain

$$
\begin{aligned}
\frac{\mathbf{1}^{+}-\boldsymbol{\Pi}_{\alpha}^{-} \mathbf{1}^{-}}{\alpha}=- & \frac{1}{\sqrt{-\boldsymbol{\mu} \boldsymbol{V} \boldsymbol{r}}} \alpha^{-1 / 2}\left(\boldsymbol{r}^{+}-\boldsymbol{\Pi}^{-} \boldsymbol{r}^{-}\right)+\frac{1}{\sqrt{-\boldsymbol{\mu V} \boldsymbol{r}}} \alpha^{-1 / 2}\left(\boldsymbol{\Pi}_{\alpha}^{-}-\boldsymbol{\Pi}^{-}\right) \boldsymbol{r}^{-} \\
& +\frac{1}{\alpha} \boldsymbol{\Xi}_{\min }^{+}\left(\alpha^{1 / 2}\right)+\frac{1}{\alpha} \boldsymbol{\Pi}_{\alpha}^{-} \boldsymbol{\Xi}_{\min }^{-}\left(\alpha^{1 / 2}\right)
\end{aligned}
$$

for sufficiently small $\alpha$.

By Theorem 3.1, (1/ $) \mathbf{\Xi}_{\min }^{+}\left(\alpha^{1 / 2}\right)$ is bounded in norm, and, by Lemma 3.5(v),

$$
\boldsymbol{\Pi}_{\alpha}^{-}-\boldsymbol{\Pi}^{-} \rightarrow \mathbf{0} \quad \text { as } \alpha \rightarrow 0
$$

Thus, it follows from (4.3) that

$$
\frac{\mathbf{1}^{+}-\boldsymbol{\Pi}_{\alpha}^{-} \mathbf{1}^{-}}{\alpha} \sim-\frac{1}{\sqrt{-\boldsymbol{\mu V \boldsymbol { r }}}} \alpha^{-1 / 2}\left(\boldsymbol{r}^{+}-\boldsymbol{\Pi}^{-} \boldsymbol{r}^{-}\right), \quad \alpha \rightarrow 0
$$

Furthermore, since

$$
\frac{\mathbf{1}-\boldsymbol{\Gamma}_{\alpha} \boldsymbol{J}_{2} \mathbf{1}}{\alpha}=\left(\begin{array}{c}
\left(\mathbf{1}^{+}-\boldsymbol{\Pi}_{\alpha}^{-} \mathbf{1}^{-}\right) / \alpha \\
\mathbf{0}
\end{array}\right) \quad \text { and } \quad \boldsymbol{J}_{1} \boldsymbol{\Gamma}_{2} \boldsymbol{r}=\left(\begin{array}{c}
\boldsymbol{r}^{+}-\boldsymbol{\Pi}^{-} \boldsymbol{r}^{-} \\
\mathbf{0}
\end{array}\right)
$$

we have

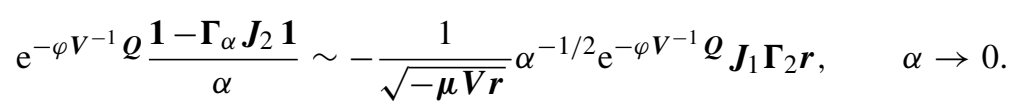

The function $\alpha \mapsto \mathrm{e}^{-\varphi \boldsymbol{V}^{-1}(\boldsymbol{Q}-\alpha \boldsymbol{I})}$ is analytic for all $\alpha$ and, by Lemma 3.5(v), $\boldsymbol{\Gamma}_{\alpha} \rightarrow \boldsymbol{\Gamma}$ as $\alpha \rightarrow 0$. Hence, the second term on the right-hand side of (4.2) is bounded for small $\alpha>0$. Therefore, for any $(e, \varphi) \in E \times(0, \infty)$,

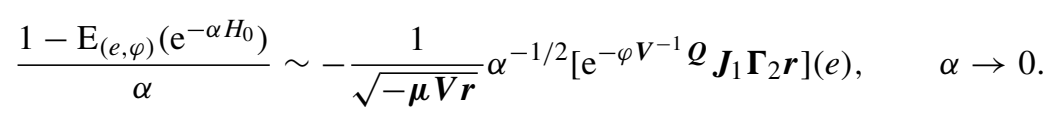

The assertion of the lemma now follows from the Tauberian theorem (see Feller (1971, Chapter XIII.5)).

(ii) We give only the sketch of the proof. For the details, see Najdanovic (2003) or refer to Jacka et al. (2005).

For any $y \in \mathbb{R}$, let the matrices $\boldsymbol{A}_{y}$ and $\boldsymbol{C}_{y}$ be components of the matrix $\mathrm{e}^{-y \boldsymbol{V}^{-1} \boldsymbol{Q}}$ given by

$$
\mathrm{e}^{-y \boldsymbol{V}^{-1} \boldsymbol{Q}}=\left(\begin{array}{ll}
\boldsymbol{A}_{y} & \boldsymbol{B}_{y} \\
\boldsymbol{C}_{y} & \boldsymbol{D}_{y}
\end{array}\right) .
$$


Then, for any $\varphi \in \mathbb{R}$,

$$
\mathrm{e}^{-\varphi \boldsymbol{V}^{-1} \boldsymbol{Q}} \boldsymbol{J}_{1} \boldsymbol{\Gamma}_{2} \boldsymbol{r}=\left(\begin{array}{c}
\boldsymbol{A}_{\varphi}\left(\boldsymbol{r}^{+}-\boldsymbol{\Pi}^{-} \boldsymbol{r}^{-}\right) \\
\boldsymbol{C}_{\varphi}\left(\boldsymbol{r}^{+}-\boldsymbol{\Pi}^{-} \boldsymbol{r}^{-}\right)
\end{array}\right)
$$

The proof of the lemma consists of first showing that the vectors $\boldsymbol{A}_{\varphi}\left(\boldsymbol{r}^{+}-\boldsymbol{\Pi}^{-} \boldsymbol{r}^{-}\right)$and $\boldsymbol{C}_{\varphi}\left(\boldsymbol{r}^{+}-\boldsymbol{\Pi}^{-} \boldsymbol{r}^{-}\right)$have the same constant sign, which implies that the function $h_{\boldsymbol{r}}$ has a constant sign. Then we deduce from part (i) that $h_{\boldsymbol{r}}$ must be positive.

By ordinary matrix algebra and the equalities $\mathrm{e}^{y \boldsymbol{V}^{-1}} \boldsymbol{Q}_{\boldsymbol{r}}=\boldsymbol{r}+y \mathbf{1}$ and $\boldsymbol{\Pi}^{-} \mathbf{1}^{-}=\mathbf{1}^{+}$, it can be shown that, for any $\varphi, y \in \mathbb{R}$, the vector $\boldsymbol{A}_{\varphi}\left(\boldsymbol{r}^{+}-\boldsymbol{\Pi}^{-} \boldsymbol{r}^{-}\right)$satisfies the equality

$$
\left(\boldsymbol{A}_{\varphi}\left(\boldsymbol{A}_{y}-\boldsymbol{\Pi}^{-} \boldsymbol{C}_{y}\right)^{-1} \boldsymbol{A}_{\varphi}^{-1}\right) \boldsymbol{A}_{\varphi}\left(\boldsymbol{r}^{+}-\boldsymbol{\Pi}^{-} \boldsymbol{r}^{-}\right)=\boldsymbol{A}_{\varphi}\left(\boldsymbol{r}^{+}-\boldsymbol{\Pi}^{-} \boldsymbol{r}^{-}\right)
$$

In addition, it can be shown that the matrix $\boldsymbol{A}_{\varphi}\left(\boldsymbol{A}_{y}-\boldsymbol{\Pi}^{-} \boldsymbol{C}_{y}\right)^{-1} \boldsymbol{A}_{\varphi}^{-1}, \varphi \neq y$, is positive and that its Perron-Frobenius eigenvalue is 1 . The last equality then implies that the vector $\boldsymbol{A}_{\varphi}\left(\boldsymbol{r}^{+}-\boldsymbol{\Pi}^{-} \boldsymbol{r}^{-}\right)$is its Perron-Frobenius eigenvector and therefore has a constant sign.

Furthermore, it can be shown that the matrix $\boldsymbol{C}_{\varphi} \boldsymbol{A}_{\varphi}^{-1}$ is positive. Hence, because

$$
\boldsymbol{C}_{\varphi}\left(\boldsymbol{r}^{+}-\boldsymbol{\Pi}^{-} \boldsymbol{r}^{-}\right)=\boldsymbol{C}_{\varphi} \boldsymbol{A}_{\varphi}^{-1} \boldsymbol{A}_{\varphi}\left(\boldsymbol{r}^{+}-\boldsymbol{\Pi}^{-} \boldsymbol{r}^{-}\right)
$$

and $\boldsymbol{A}_{\varphi}\left(\boldsymbol{r}^{+}-\boldsymbol{\Pi}^{-} \boldsymbol{r}^{-}\right)$has a constant sign, we deduce that the vector $\boldsymbol{C}_{\varphi}\left(\boldsymbol{r}^{+}-\boldsymbol{\Pi}^{-} \boldsymbol{r}^{-}\right)$has the same constant sign. Thus, the function $h_{r}$ has a constant sign and, since $\mathrm{P}_{(e, \varphi)}\left(H_{0}>t\right)>0$, it follows from part (i) that the function $h_{\boldsymbol{r}}$ is positive.

For the proof of Theorem 2.1 we need two more lemmas.

Lemma 4.2. (i) Let $\left\{f_{n}, n \in \mathbb{N}\right\}$ and $f$ be nonnegative random variables, on a probability space $(\Omega, \mathcal{F}, \mathrm{P})$, such that $\mathrm{E} f_{n}=\mathrm{E} f=1$, where expectation is taken with respect to the probability measure $\mathrm{P}$. If $f_{n} \rightarrow f$ almost surely as $n \rightarrow \infty$, then $f_{n} \rightarrow f$ in $L^{1}(\Omega, \mathcal{F}, \mathrm{P})$ as $n \rightarrow \infty$.

(ii) Let $\left\{\mathrm{P}_{n}, n \in \mathbb{N}\right\}$ and $\mathrm{P}$ be probability measures, on a measurable space $(\Omega, \mathcal{F})$, such that, for any $A \in \mathcal{F}, \mathrm{P}_{n}(A) \rightarrow \mathrm{P}(A)$ as $n \rightarrow \infty$. Then the measures $\left\{\mathrm{P}_{n}, n \in \mathbb{N}\right\}$ converge weakly to $\mathrm{P}$ on $\mathcal{F}$.

Proof. (i) Since $\left\{f_{n}, n \in \mathbb{N}\right\}$ and $f$ are nonnegative and $\mathrm{E} f_{n}=\mathrm{E} f=1$, the functions $\left\{f_{n}(\omega), n \in \mathbb{N}\right\}$ and $f(\omega), \omega \in \Omega$, are densities with respect to the measure P. In addition, $f_{n} \rightarrow f$ almost surely as $n \rightarrow \infty$ and, so, $f_{n} \rightarrow f$ in probability as $n \rightarrow \infty$. Therefore, by Theorem 2.2 of Jacka and Roberts (1997), $f_{n} \rightarrow f$ in $L^{1}(\Omega, \mathcal{F}, \mathrm{P})$ as $n \rightarrow \infty$.

(ii) For any $A \in \mathcal{F}$, let $\mathrm{P}_{n}(A) \rightarrow \mathrm{P}(A)$ as $n \rightarrow \infty$. Then, by the definition of strong convergence in Jacka and Roberts (1997), the measures $\left\{\mathrm{P}_{n}, n \in \mathbb{N}\right\}$ converge strongly to $\mathrm{P}$, which, by Theorem 2.1 of Jacka and Roberts (1997), implies that the measures $\left\{\mathrm{P}_{n}, n \in \mathbb{N}\right\}$ converge weakly to $\mathrm{P}$.

Lemma 4.3. The function $h_{\boldsymbol{r}}(e, \varphi)$ is harmonic for the process $\left(X_{t}, \varphi_{t}\right)_{t \geq 0}$, and the process $\left(h_{\boldsymbol{r}}\left(X_{t}, \varphi_{t}\right) 1\left(t<H_{0}\right)\right)_{t \geq 0}$ is a martingale under $\mathrm{P}_{(e, \varphi)}$.

Proof. The function $h_{\boldsymbol{r}}$ is continuously differentiable in $\varphi$, which, by (3.4), implies that $h_{\boldsymbol{r}}$ is in the domain of the infinitesimal generator $g$ of the process $\left(X_{t}, \varphi_{t}\right)_{t \geq 0}$ and that 
$g h_{r}=0$. Hence, the function $h_{r}(e, \varphi)$ is harmonic for the process $\left(X_{t}, \varphi_{t}\right)_{t \geq 0}$ and the process $\left(h_{\boldsymbol{r}}\left(X_{t}, \varphi_{t}\right)\right)_{t \geq 0}$ is a local martingale under $\mathrm{P}_{(e, \varphi)}$. It follows that the process

$$
\left(h_{\boldsymbol{r}}\left(X_{t \wedge H_{0}}, \varphi_{t \wedge H_{0}}\right)=h_{\boldsymbol{r}}\left(X_{t}, \varphi_{t}\right) 1\left(t<H_{0}\right)\right)_{t \geq 0}
$$

is also a local martingale under $\mathrm{P}_{(e, \varphi)}$ (the equality of the processes is valid because

$$
h_{r}\left(X_{H_{0}}, \varphi_{H_{0}}\right)=0
$$

if the process $\left(X_{t}, \varphi_{t}\right)_{t \geq 0}$ starts in $\left.E_{0}^{+}\right)$. Since the process $\left(h_{r}\left(X_{t}, \varphi_{t}\right) 1\left(t<H_{0}\right)\right)_{t \geq 0}$ is bounded on every finite interval, it follows that it is a martingale under $\mathrm{P}_{(e, \varphi)}$.

Proof of Theorem 2.1. By Lemmas 4.1(ii) and 4.3, the function $h_{\boldsymbol{r}}(e, \varphi)$ is positive and harmonic for the process $\left(X_{t}, \varphi_{t}\right)_{t \geq 0}$. Therefore, the measure $\mathrm{P}_{(e, \varphi)}^{h_{r}}$ is well defined.

For a fixed $(e, \varphi) \in E_{0}^{+}$and $t \geq 0$, and any $T \geq 0$, let $Z_{T}$ be a random variable defined by

$$
Z_{T}=\frac{\mathrm{P}_{\left(X_{t}, \varphi_{t}\right)}\left(H_{0}>T-t\right)}{\mathrm{P}_{(e, \varphi)}\left(H_{0}>T\right)} 1\left(t<H_{0}\right) .
$$

Then, by Lemmas $4.1,4.2$, and 4.3 , the random variables $Z_{T}$ converge in $L^{1}\left(\Omega, \mathcal{F}, \mathrm{P}_{(e, \varphi)}\right)$, as $T \rightarrow \infty$, to the random variable $\left(h_{\boldsymbol{r}}\left(X_{t}, \varphi_{t}\right) / h_{\boldsymbol{r}}(e, \varphi)\right) 1\left(t<H_{0}\right)$. Therefore, by (4.1), for fixed $t \geq 0$ and $A \in \mathcal{F}_{t}$, we have

$$
\begin{aligned}
\lim _{T \rightarrow \infty} \mathrm{P}_{(e, \varphi)}^{(T)}(A) & =\lim _{T \rightarrow \infty} \mathrm{E}_{(e, \varphi)}\left(1(A) Z_{T}\right) \\
& =\mathrm{E}_{(e, \varphi)}\left(1(A) \frac{h_{r}\left(X_{t}, \varphi_{t}\right)}{h_{r}(e, \varphi)} 1\left(t<H_{0}\right)\right) \\
& =\mathrm{P}_{(e, \varphi)}^{h_{r}}(A),
\end{aligned}
$$

which, by Lemma 4.2 (ii), implies that the restrictions of the measures $\left(\mathrm{P}_{(e, \varphi)}^{(T)}\right)_{y \geq 0}$ to $\mathcal{F}_{t}$ converge weakly to the restriction of $\mathrm{P}_{(e, \varphi)}^{h_{r}}$ as $T \rightarrow \infty$.

\section{The negative-drift case: proof of Theorem 2.2}

We again start by considering $\lim _{T \rightarrow \infty} \mathrm{P}_{(e, \varphi)}^{(T)}(A)$ for $A \in \mathcal{F}_{t}$. As in the oscillating case, we need to find

$$
\lim _{T \rightarrow \infty} \frac{\mathrm{P}_{\left(e^{\prime}, \varphi^{\prime}\right)}\left(H_{0}>T-t\right)}{\mathrm{P}_{(e, \varphi)}\left(H_{0}>T\right)} .
$$

We recall that $\beta_{0}$ denotes the point at which the Perron-Frobenius eigenvalue $\alpha(\beta)$ of the matrix $\boldsymbol{Q}-\beta \boldsymbol{V}$ attains its global minimum (see Lemma 3.9), that $\alpha_{0}=\alpha\left(\beta_{0}\right)$ and $\boldsymbol{g}_{0}$ denote the Perron-Frobenius eigenvalue and right eigenvector, respectively, of the matrix $\boldsymbol{Q}-\beta_{0} \boldsymbol{V}$, and that $\boldsymbol{G}_{0}$ denotes the diagonal matrix $\operatorname{diag}\left(\boldsymbol{g}_{0}\right)$. We also recall the $|E| \times|E|$ matrix $\boldsymbol{Q}^{0}$, given by (2.1) as

$$
Q^{0}\left(e, e^{\prime}\right)=\left[\boldsymbol{G}_{0}^{-1}\left(\boldsymbol{Q}-\alpha_{0} \boldsymbol{I}-\beta_{0} \boldsymbol{V}\right) \boldsymbol{G}_{0}\right]\left(e, e^{\prime}\right) .
$$

By Lemma 3.11, the matrix $\boldsymbol{Q}^{0}$ is a conservative irreducible $Q$-matrix. Let $\boldsymbol{V}^{-1} \boldsymbol{Q} \boldsymbol{\Gamma}^{0}=\boldsymbol{\Gamma}^{0} \boldsymbol{G}^{0}$ be the unique Wiener-Hopf factorization of the matrix $V^{-1} Q^{0}$, and let $\Gamma_{2}^{0}=J \Gamma^{0} J$. 
Our aim is to prove the following lemma.

Lemma 5.1. (i) The function $h_{\boldsymbol{r}^{0}}(e, \varphi, t)$ is given by

$$
h_{\boldsymbol{r}^{0}}(e, \varphi, t) \equiv-\mathrm{e}^{-\alpha_{0} t} \mathrm{e}^{-\beta_{0} \varphi}\left[\boldsymbol{G}_{0} \mathrm{e}^{-\varphi \boldsymbol{V}^{-1}} \boldsymbol{Q}^{0} \boldsymbol{J}_{1} \boldsymbol{\Gamma}_{2}^{0} \boldsymbol{r}^{0}\right](e)>0, \quad(e, \varphi, t) \in E_{0}^{+} \times[0, \infty) .
$$

(ii) If

$$
\lim _{T \rightarrow \infty} \frac{\mathrm{P}_{\left(e^{\prime}, \varphi^{\prime}\right)}\left(H_{0}>T-t\right)}{\mathrm{P}_{(e, \varphi)}\left(H_{0}>T\right)} \quad \text { exists it is equal to } \frac{h_{\boldsymbol{r}^{0}}\left(e^{\prime}, \varphi^{\prime}, t\right)}{h_{\boldsymbol{r}^{0}}(e, \varphi, 0)} .
$$

To prove this, we will need some auxiliary lemmas. For $\alpha>0$, let $\boldsymbol{V}^{-1}\left(\boldsymbol{Q}^{0}-\alpha \boldsymbol{I}\right) \boldsymbol{\Gamma}_{\alpha}^{0}=$ $\boldsymbol{\Gamma}_{\alpha}^{0} \boldsymbol{G}_{\alpha}^{0}$ be the unique Wiener-Hopf factorization of the matrix $\boldsymbol{V}^{-1}\left(\boldsymbol{Q}^{0}-\alpha \boldsymbol{I}\right)$ and, for a fixed $(e, \varphi) \in E \times \mathbb{R}$, let a function $L_{(e, \varphi)}(\alpha), \alpha \geq \alpha_{0}$, be defined by

$$
L_{(e, \varphi)}(\alpha)=\left[\frac{\mathbf{1}-\mathrm{e}^{-\beta_{0} \varphi} \boldsymbol{G}_{0} \mathrm{e}^{-\varphi \boldsymbol{V}^{-1}\left(\boldsymbol{Q}^{0}-\left(\alpha-\alpha_{0}\right) \boldsymbol{I}\right)} \boldsymbol{\Gamma}_{\alpha-\alpha_{0}}^{0} \boldsymbol{G}_{0}^{-1} \boldsymbol{J}_{2} \mathbf{1}}{\alpha}\right](e) .
$$

By Lemmas 3.3 and 3.12, for $\alpha>0$ we have

$$
\begin{aligned}
L_{(e, \varphi)}(\alpha)=\left[\frac{\mathbf{1}-\mathrm{e}^{-\varphi \boldsymbol{V}^{-1}(\boldsymbol{Q}-\alpha \boldsymbol{I})} \boldsymbol{\Gamma}_{\alpha} \boldsymbol{J}_{2} \mathbf{1}}{\alpha}\right](e) & =\frac{1-\mathrm{E}_{(e, \varphi)}\left(\mathrm{e}^{-\alpha H_{0}}\right)}{\alpha} \\
& =\int_{0}^{\infty} \mathrm{e}^{-\alpha t} \mathrm{P}_{(e, \varphi)}\left(H_{0}>t\right) \mathrm{d} t .
\end{aligned}
$$

Lemma 5.2. For any $(e, \varphi) \in E_{0}^{+}$, the function $L_{(e, \varphi)}(\alpha)$ is analytic for $\operatorname{Re}(\alpha)>\alpha_{0}$.

Proof. By the definition in Lemma 3.3, the matrices $\Pi_{\alpha}^{+}$and $\Pi_{\alpha}^{-}$are analytic for $\operatorname{Re}(\alpha)>0$. Hence, the matrix $\boldsymbol{\Gamma}_{\alpha}$ is analytic for $\operatorname{Re}(\alpha)>0$ and, therefore, by Lemma 3.12, the matrix $\Gamma_{\alpha-\alpha_{0}}^{0}$ is analytic for $\operatorname{Re}(\alpha)>\alpha_{0}$. It follows that the numerator of $L_{(e, \varphi)}(\alpha)$ in (5.1) is analytic for $\operatorname{Re}(\alpha)>\alpha_{0}$ and, since

$$
\mathrm{e}^{-\beta_{0} \varphi} \boldsymbol{G}_{0} \mathrm{e}^{-\varphi \boldsymbol{V}^{-1}\left(\boldsymbol{Q}^{0}+\alpha_{0} \boldsymbol{I}\right)} \boldsymbol{\Gamma}_{-\alpha_{0}}^{0} \boldsymbol{G}_{0}^{-1} \boldsymbol{J}_{2} \mathbf{1}=\mathrm{e}^{-\varphi \boldsymbol{V}^{-1} \boldsymbol{Q}} \boldsymbol{\Gamma} \boldsymbol{J}_{2} \mathbf{1}=\mathbf{1},
$$

the numerator of $L_{(e, \varphi)}(\alpha)$ vanishes for $\alpha=0$. Therefore, $L_{(e, \varphi)}(\alpha)$ is analytic for $\operatorname{Re}(\alpha)>\alpha_{0}$.

We adopt the convention that objects (e.g. vectors and matrices) with the superscript ' 0 ' are associated with the matrix $\boldsymbol{Q}^{0}$ and are defined in the same way as their counterparts associated with the matrix $Q$.

Lemma 5.3. Let all nonzero eigenvalues of the matrix $\boldsymbol{V}^{-1} Q^{0}$ be simple. Then, for some nonzero constant $c$,

(i) $\left(\boldsymbol{\Gamma}_{\alpha-\alpha_{0}}^{0}-\boldsymbol{\Gamma}^{0}\right) \boldsymbol{G}_{0}^{-1} \boldsymbol{J}_{2} \mathbf{1} \sim c\left(\alpha-\alpha_{0}\right)^{1 / 2} \boldsymbol{J}_{1} \boldsymbol{\Gamma}_{2}^{0} \boldsymbol{r}^{0}$ as $\alpha \rightarrow \alpha_{0}$,

(ii) $L_{(e, \varphi)}(\alpha)-L_{(e, \varphi)}\left(\alpha_{0}\right) \sim c\left(\alpha-\alpha_{0}\right)^{1 / 2} \mathrm{e}^{-\beta_{0} \varphi}\left[\boldsymbol{G}_{0} \mathrm{e}^{-\varphi \boldsymbol{V}^{-1}} \boldsymbol{Q}^{0} \boldsymbol{J}_{1} \boldsymbol{\Gamma}_{2}^{0} \boldsymbol{r}^{0}\right](e)$ as $\alpha \rightarrow \alpha_{0}$.

Proof. (i) Let $\boldsymbol{g}^{-}$be a nonnegative vector on $E^{-}$. Then

$$
\boldsymbol{g}^{-}=\sum_{k=1}^{m} a_{k} \boldsymbol{g}_{k}^{0,-}
$$


for some constants $a_{k}, k=1, \ldots, m$, where the vectors $\boldsymbol{g}_{k}^{0,-}, k=1, \ldots, m$, form a basis in the space of all vectors on $E^{-}$and are associated with the eigenvalues of the matrix $G^{0,-}$. By Lemma 3.6(ii), the constant $a_{\mathrm{min}}$, which is associated with $\mathbf{g}_{\min }^{0,-}=\mathbf{1}^{-}$in the previous linear combination, is not 0 . Thus,

$$
\boldsymbol{\Pi}_{\alpha}^{0,-} \boldsymbol{g}^{-}=a_{\min } \boldsymbol{\Pi}_{\alpha}^{0,-} \mathbf{1}^{-}+\sum_{\left\{k=1, \ldots, m: \boldsymbol{g}_{k}^{0,-} \neq \boldsymbol{g}_{\min }^{0,-}\right\}} a_{k} \boldsymbol{\Pi}_{\alpha}^{0,-} \boldsymbol{g}_{k}^{0,-} .
$$

By (3.6) and Lemma 3.11, the matrices $\boldsymbol{Q}^{0}$ and $\boldsymbol{V}$ define the oscillating case. Therefore, by (4.4),

$$
\mathbf{1}^{+}-\boldsymbol{\Pi}_{\alpha}^{0,-} \mathbf{1}^{-} \sim-\frac{1}{\sqrt{-\boldsymbol{\mu}^{0} \boldsymbol{V} \boldsymbol{r}^{0}}} \alpha^{1 / 2}\left(\boldsymbol{r}^{0,+}-\boldsymbol{\Pi}^{0,-} \boldsymbol{r}^{0,-}\right), \quad \alpha \rightarrow 0
$$

We must also determine the behaviour of $\boldsymbol{\Pi}_{\alpha}^{0,-} \boldsymbol{g}_{k}^{0,-}, k=1, \ldots, m$ (with $\boldsymbol{g}_{k}^{0,-} \neq \boldsymbol{g}_{\min }^{0,-}$ ). Since, by assumption, all nonzero eigenvalues of the matrix $\boldsymbol{V}^{-1} \boldsymbol{Q}^{0}$ are simple, it can be shown (see Wilkinson (1965)) that there exist vectors $\boldsymbol{v}_{k, n}, n \in \mathbb{N}$, on $E$ such that

$$
\boldsymbol{\Pi}_{\alpha}^{0,-} \boldsymbol{g}_{k}^{0,-}-\boldsymbol{\Pi}^{0,-} \boldsymbol{g}_{k}^{0,-}=\sum_{n=1}^{\infty} \alpha^{n}\left(\boldsymbol{v}_{k, n}^{+}-\boldsymbol{\Pi}_{\alpha}^{0,-} \boldsymbol{v}_{k, n}^{-}\right) .
$$

From (5.3), (5.4), and (5.5), and because, by Lemma 3.5(v), $\Pi_{\alpha}^{0,-} \rightarrow \Pi^{0,-}$ as $\alpha \rightarrow 0$, we find that

$$
\boldsymbol{\Pi}_{\alpha}^{0,-} \boldsymbol{g}^{-}-\boldsymbol{\Pi}^{0,-} \boldsymbol{g}^{-} \sim-\frac{a_{\mathrm{min}}}{\sqrt{-\boldsymbol{\mu}^{0} \boldsymbol{V} \boldsymbol{r}^{0}}} \alpha^{1 / 2}\left(\boldsymbol{r}^{0,+}-\boldsymbol{\Pi}^{0,-} \boldsymbol{r}^{0,-}\right), \quad \alpha \rightarrow 0,
$$

which proves part (i).

(ii) By the definition of $L_{(e, \varphi)}(\alpha)$,

$$
\begin{aligned}
& L_{(e, \varphi)}(\alpha)-L_{(e, \varphi)}\left(\alpha_{0}\right) \\
& =-\left[\frac{\left(\alpha-\alpha_{0}\right)\left(\mathbf{1}-\mathrm{e}^{-\beta_{0} \varphi} \boldsymbol{G}_{0} \mathrm{e}^{-\varphi \boldsymbol{V}^{-1} \boldsymbol{Q}^{0}} \boldsymbol{\Gamma}^{0} \boldsymbol{G}_{0}^{-1} \boldsymbol{J}_{2} \mathbf{1}\right)}{\alpha \alpha_{0}}\right. \\
& +\frac{\alpha_{0}\left(\mathrm{e}^{-\beta_{0} \varphi} \boldsymbol{G}_{0}\left(\mathrm{e}^{-\varphi \boldsymbol{V}^{-1}\left(\boldsymbol{Q}^{0}-\left(\alpha-\alpha_{0}\right)\right)}-\mathrm{e}^{-\varphi \boldsymbol{V}^{-1}} \boldsymbol{Q}^{0}\right) \boldsymbol{\Gamma}^{0} \boldsymbol{G}_{0}^{-1} \boldsymbol{J}_{2} \mathbf{1}\right)}{\alpha \alpha_{0}} \\
& +\frac{\mathrm{e}^{-\beta_{0} \varphi} \boldsymbol{G}_{0} \mathrm{e}^{-\varphi \boldsymbol{V}^{-1}} \boldsymbol{Q}^{0}\left(\boldsymbol{\Gamma}_{\alpha-\alpha_{0}}^{0}-\boldsymbol{\Gamma}^{0}\right) \boldsymbol{G}_{0}^{-1} \boldsymbol{J}_{2} \mathbf{1}}{\alpha} \\
& \left.+\frac{\mathrm{e}^{-\beta_{0} \varphi} \boldsymbol{G}_{0}\left(\mathrm{e}^{-\varphi \boldsymbol{V}^{-1}\left(\boldsymbol{Q}^{0}-\left(\alpha-\alpha_{0}\right)\right)}-\mathrm{e}^{-\varphi \boldsymbol{V}^{-1} \boldsymbol{Q}^{0}}\right)\left(\boldsymbol{\Gamma}_{\alpha-\alpha_{0}}^{0}-\boldsymbol{\Gamma}^{0}\right) \boldsymbol{G}_{0}^{-1} \boldsymbol{J}_{2} \mathbf{1}}{\alpha}\right](e) .
\end{aligned}
$$

The function $\alpha \mapsto \mathrm{e}^{-\varphi V^{-1}\left(Q^{0}-\left(\alpha-\alpha_{0}\right)\right)}$ is analytic for all $\alpha$, which implies that

$$
\mathrm{e}^{-\varphi \boldsymbol{V}^{-1}\left(\boldsymbol{Q}^{0}-\left(\alpha-\alpha_{0}\right)\right)}-\mathrm{e}^{-\varphi \boldsymbol{V}^{-1} \boldsymbol{Q}^{0}}
$$

tends to 0 as $\alpha \rightarrow \alpha_{0}$. Hence, by part (i) and the last equality, part (ii) holds.

Lemma 5.4. For a fixed $(e, \varphi) \in E_{0}^{+}$, the function $L_{(e, \varphi)}\left(\alpha+\alpha_{0}\right), \alpha>0$, is the Laplace transform of $\mathrm{e}^{-\alpha_{0} t} \mathrm{P}_{(e, \varphi)}\left(H_{0}>t\right)$. 
Proof. By (5.2), $L_{(e, \varphi)}(\alpha), \alpha>0$, is a Laplace transform and, therefore, by Theorem 1a of Feller (1971, Chapter XIII.4), is completely monotone for $\alpha \geq 0$. In addition, by Lemma 5.2, $L_{(e, \varphi)}(\alpha)$ is analytic for $\alpha>\alpha_{0}$. Since the analytic continuation of a completely monotone function is completely monotone, it follows that $L_{(e, \varphi)}(\alpha)$ is completely monotone for $\alpha>\alpha_{0}$ and, therefore, is a Laplace transform of some measure on $[0, \infty)$. By the uniqueness of the inverse of the Laplace transform, it follows from (5.2) that, for $\alpha>0, L_{(e, \varphi)}\left(\alpha+\alpha_{0}\right)$ is the Laplace transform of $\mathrm{e}^{-\alpha_{0} t} \mathrm{P}_{(e, \varphi)}\left(H_{0}>t\right)$.

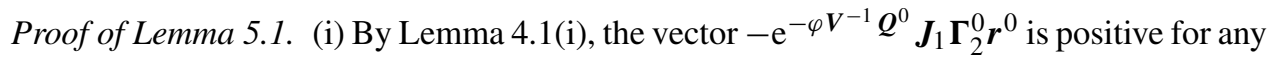
$\varphi \in \mathbb{R}$. Since the matrix $\boldsymbol{G}_{0}$ is positive by definition, it follows that the function $h_{\boldsymbol{r}^{0}}(e, \varphi, t)$ is positive for any $(e, \varphi, t) \in E_{0}^{+} \times[0, \infty)$.

(ii) By Lemma 5.4,

$$
\frac{L_{(e, \varphi)}\left(\alpha+\alpha_{0}\right)}{\alpha}-\frac{L_{(e, \varphi)}\left(\alpha_{0}\right)}{\alpha}
$$

is the Laplace transform of the monotone function

$$
t \mapsto \int_{0}^{t} \mathrm{e}^{-\alpha_{0} s} \mathrm{P}_{(e, \varphi)}\left(H_{0}>s\right) \mathrm{d} s-L_{(e, \varphi)}\left(\alpha_{0}\right) .
$$

Therefore, by the Tauberian theorem (see Feller (1971, Chapter XIII.5)),

$$
\int_{0}^{t} \mathrm{e}^{-\alpha_{0} s} \mathrm{P}_{(e, \varphi)}\left(H_{0}>s\right) \mathrm{d} s-L_{(e, \varphi)}\left(\alpha_{0}\right) \sim \frac{c}{\Gamma\left(\frac{1}{2}\right)} t^{-1 / 2} \mathrm{e}^{-\beta_{0} \varphi}\left[\boldsymbol{G}_{0} \mathrm{e}^{-\varphi \boldsymbol{V}^{-1} \boldsymbol{Q}^{0}} \boldsymbol{J}_{1} \boldsymbol{\Gamma}_{2}^{0} \boldsymbol{r}^{0}\right](e)
$$

as $t \rightarrow \infty$. Then, for fixed $(e, \varphi),\left(e^{\prime}, \varphi^{\prime}\right) \in E_{0}^{+}$,

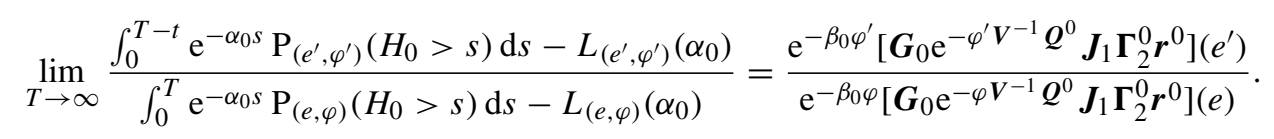

The statement in the lemma is now proved since, by l'Hôpital's rule,

$$
\lim _{T \rightarrow \infty} \frac{\int_{0}^{T-t} \mathrm{e}^{-\alpha_{0} s} \mathrm{P}_{\left(e^{\prime}, \varphi^{\prime}\right)}\left(H_{0}>s\right) \mathrm{d} s-L_{\left(e^{\prime}, \varphi^{\prime}\right)}\left(\alpha_{0}\right)}{\int_{0}^{T} \mathrm{e}^{-\alpha_{0} s} \mathrm{P}_{(e, \varphi)}\left(H_{0}>s\right) \mathrm{d} s-L_{(e, \varphi)}\left(\alpha_{0}\right)}=\mathrm{e}^{\alpha_{0} t} \lim _{T \rightarrow \infty} \frac{\mathrm{P}_{\left(e^{\prime}, \varphi^{\prime}\right)}\left(H_{0}>T-t\right)}{\mathrm{P}_{(e, \varphi)}\left(H_{0}>T\right)},
$$

if the latter limit exists.

Lemma 5.5. The function $h_{\boldsymbol{r}^{0}}$ is space-time harmonic for the process $\left(X_{t}, \varphi_{t}\right)_{t \geq 0}$, and the process $\left(h_{\boldsymbol{r}^{0}}\left(X_{t}, \varphi_{t}, t\right) 1\left(t<H_{0}\right)\right)_{t \geq 0}$ is a martingale under $\mathrm{P}_{(e, \varphi)}$.

Proof. The function $h_{\boldsymbol{r}^{0}}$ is continuously differentiable in $\varphi$ and $t$, which, by (3.5), implies that it is in the domain of the infinitesimal generator $\mathcal{A}$ of the process $\left(X_{t}, \varphi_{t}\right)_{t \geq 0}$, and that $\mathcal{A} h_{\boldsymbol{r}^{0}}=0$. Hence, the function $h_{\boldsymbol{r}^{0}}(e, \varphi, t)$ is space-time harmonic for the process $\left(X_{t}, \varphi_{t}\right)_{t \geq 0}$ and the process $\left(h_{r^{0}}\left(X_{t}, \varphi_{t}, t\right)\right)_{t \geq 0}$ is a local martingale under $\mathrm{P}_{(e, \varphi)}$. It follows that the process $\left(h_{\boldsymbol{r}^{0}}\left(X_{t \wedge H_{0}}, \varphi_{t \wedge H_{0}}, t \wedge H_{0}\right)=h_{\boldsymbol{r}^{0}}\left(X_{t}, \varphi_{t}, t\right) 1\left(t<H_{0}\right)\right)_{t \geq 0}$ is also a local martingale under $\mathrm{P}_{(e, \varphi)}$. Since the process $\left(h_{\boldsymbol{r}^{0}}\left(X_{t}, \varphi_{t}, t\right) 1\left(t<H_{0}\right)\right)_{t \geq 0}$ is bounded on every finite interval, it follows that it is a martingale under $\mathrm{P}_{(e, \varphi)}$. 
Proof of Theorem 2.2. By Lemmas 5.1(i) and 5.5, the function $h_{r^{0}}(e, \varphi, t)$ is positive and space-time harmonic for the process $\left(X_{t}, \varphi_{t}\right)_{t \geq 0}$. The measure $\mathrm{P}_{(e, \varphi)}^{h^{0}}$ is therefore well defined.

For a fixed $(e, \varphi) \in E_{0}^{+}$and $t \geq 0$, and any $T \geq 0$, let $Z_{T}$ be a random variable defined by

$$
Z_{T}=\frac{\mathrm{P}_{\left(X_{t}, \varphi_{t}\right)}\left(H_{0}>T-t\right)}{\mathrm{P}_{(e, \varphi)}\left(H_{0}>T\right)} 1\left(t<H_{0}\right) .
$$

Then, by Lemmas 5.1, 4.2(i), and 5.5, the random variable $Z_{T}$ converges to

$$
\frac{h_{\boldsymbol{r}^{0}}\left(X_{t}, \varphi_{t}\right)}{h_{\boldsymbol{r}^{0}}(e, \varphi)} 1\left(t<H_{0}\right)
$$

in $L^{1}\left(\Omega, \mathcal{F}, \mathrm{P}_{(e, \varphi)}\right)$ as $T \rightarrow \infty$. Therefore, by (4.1), for a fixed $t \geq 0$ and $A \in \mathcal{F}_{t}$,

$$
\lim _{T \rightarrow \infty} \mathrm{P}_{(e, \varphi)}^{(T)}(A)=\lim _{T \rightarrow \infty} \mathrm{E}_{(e, \varphi)}\left(1(A) Z_{T}\right)=\mathrm{P}_{(e, \varphi)}^{h^{0}}(A),
$$

which, by Lemma 4.2(ii), implies that the restrictions to $\mathcal{F}_{t}$ of the measures $\left(\mathrm{P}_{(e, \varphi)}^{(T)}\right)_{y \geq 0}$ converge weakly to the restriction to $\mathcal{F}_{t}$ of $\mathrm{P}_{(e, \varphi)}^{h^{0}}$ as $T \rightarrow \infty$.

\section{References}

Barlow, M. T., Rogers, L. C. G. ANd Williams, D. (1980). Wiener-Hopf factorization for matrices. In Seminar on Probability XIV (Lecture Notes Math. 784), Springer, Berlin, pp. 324-331.

Bertoin, J. And Doney, R. A. (1994). On conditioning a random walk to stay nonnegative. Ann. Prob. 22, $2152-2167$.

Cohen, J. C. (1981). Convexity of the dominant eigenvalue of an essentially nonnegative matrix. Proc. Amer. Math. Soc. 81, 656-658.

Feller, W. (1971). An Introduction to Probability Theory and Its Applications, Vol. 2. John Wiley, New York.

Iglehart, D. L. (1974). Random walks with negative drift conditioned to stay positive. J. Appl. Prob. 11, 742-751.

JACKA, S. D. AND Roberts, G. O. (1988). Conditional diffusions: their infinitesimal generators and limit laws. Res. Rep., Department of Statistics, University of Warwick.

JaCKa, S. D. And Roberts, G. O. (1997). On strong forms of weak convergence. Stoch. Process. Appl. 67, 41-53.

JACKA, S. D. AND WARREN, J. (2002). Examples of convergence and non-convergence of Markov chains conditioned not to die. Electron. J. Prob. 7, 22 pp.

JaCKa, S. D., LAZIC, Z. AND Warren, J. (2005). Conditioning an additive functional of a Markov chain to stay nonnegative. II. Hitting a high level. Adv. Appl. Prob. 37, 1035-1055.

Knight, F. B. (1969). Brownian local times and taboo processes. Trans. Amer. Math. Soc. 143, 173-185.

NaJdAnovic, Z. (2003). Conditioning a Markov chain upon the behaviour of an additive functional. Doctoral Thesis, University of Warwick.

PINSKY, R. G. (1985). On the convergence of diffusion processes conditioned to remain in a bounded region for large time to limiting positive recurrent diffusion processes. Ann. Prob. 13, 363-378.

Seneta, E. (1981). Nonnegative Matrices and Markov Chains, 2nd edn. Springer, New York.

Wilkinson, J. H. (1965). The Algebraic Eigenvalue Problem. Clarendon Press, Oxford. 